\title{
Nuclear magnetic resonance relaxometry of water in two and quasi-two dimensions
}

\author{
D. A. Faux, ${ }^{1}$ P. J. McDonald, ${ }^{1}$ N. C. Howlett, ${ }^{1}$ J. S. Bhatt, ${ }^{1}$ and S. V. Churakov ${ }^{2}$ \\ ${ }^{1}$ Physics Department, University of Surrey, Guildford, GU2 7XH, United Kingdom \\ ${ }^{2}$ Paul Scherrer Institute, Laboratory of Waste Management, CH-5232 Villigen, Switzerland
}

(Received 8 February 2013; published 17 June 2013)

\begin{abstract}
Molecular dynamics (MD) and Monte Carlo (MC) methods are used to determine the spin-pair correlation function $G^{*}(t)$ for the diffusion of bulk water in three dimensions (3D) and pore water in two dimensions (2D) and quasi-two dimensions (Q2D). The correlation function is required for the determination of the nuclear magnetic resonance spin-lattice and spin-spin relaxation times $T_{1}$ and $T_{2}$. It is shown that the analytic form of the powder-average correlation function, introduced by Sholl [Sholl, J. Phys. C: Solid State Phys. 7, 3378 (1974)] for the diffusion of spins on a 3D lattice, is of general validity. An analytic expression for $G^{*}(t)$ for a uniform spin fluid is derived in 2D. An analytic expression for the long-time behavior of $G^{*}(t)$ is derived for spins diffusing on 3D, 2D, and Q2D lattices. An analytic correction term, which accounts for spin pairs outside the scope of the numerical simulations, is derived for $3 \mathrm{D}$ and $2 \mathrm{D}$ and shown to improve the accuracy of the simulations. The contributions to $T_{1}$ due to translational and rotational motion obtained from the MD simulation of bulk water at $300 \mathrm{~K}$ are $7.4 \mathrm{~s}$ and $10 \pm 1 \mathrm{~s}$, respectively, at $150 \mathrm{MHz}$, leading to an overall time of $4.3 \pm 0.4 \mathrm{~s}$ compared to the experimental value of $3.8 \mathrm{~s}$. In Q2D systems, in which water is confined by alpha-quartz surfaces to thicknesses of 1-5 nm, $T_{1}$ for both translational and rotational relaxation is reduced due to the orientation and adsorption of spins at the surfaces. A method of parametrizing the MC lattice-diffusion simulations in 3D, 2D, and Q2D systems is presented. MC results for $G^{*}(t)$ for 3D and 2D systems are found to be consistent with an analytic uniform fluid model for $t \gtrsim 40 \mathrm{ps}$. The value of $T_{1}$ for translational diffusion obtained from the MC simulation of bulk water is found to be $4.8 \mathrm{~s}$ at $15 \mathrm{MHz} . G^{*}(t)$ obtained from MC simulations of Q2D systems, where water is confined by hard walls, is found to execute a distinct transition from $3 \mathrm{D}$ to $2 \mathrm{D}$ behavior. The $T_{1}$ is found to be similar to the $3 \mathrm{D}$ bulk water result at all pore thicknesses.
\end{abstract}

DOI: 10.1103/PhysRevE.87.062309

PACS number(s): 76.60.-k, 66.30.Pa, 47.11.Mn, 61.20.Ja

\section{INTRODUCTION}

The dynamics of fluids in confined geometries is of interest from multiple perspectives. The fluid dynamics may be important in its own right for material functionality, for instance, in the case of molecular sieves, membrane science, and catalysis. Alternatively, the dynamics may be of interest for fluid extraction, for instance, in reservoir rocks, food processing, and the curing of coatings. Finally, the fluid dynamics may be important as a probe of the morphology of the confining matrix. All three currently apply to the case of cement-based materials, which inspired this study [1]. The mechanical properties of concrete depend in part on the porosity; degradation processes are dependent on repeated cycles of water ingress and egress and the nanoscale morphology of calcium-silicate hydrates, the active component of cement, remains a topic of active debate.

Time-domain nuclear magnetic resonance (NMR) of fluids in porous media provides a powerful means of characterizing porous media [2,3]. For microscale to mesoscale porous systems, NMR pulsed-field-gradient diffusometry is particularly powerful. For nanoscale to microscale systems, NMR relaxometry is generally more useful. NMR relaxometry measures the nuclear spin-lattice $\left(T_{1}\right)$ and spin-spin $\left(T_{2}\right)$ relaxation times of the, usually, ${ }^{1} \mathrm{H}$ nuclei (i.e., magnetic "spins") of the fluid, usually water or oil. In general terms, the relaxation times of fluid molecules adsorbed to the surface or encountering the surface through diffusion are very different compared to those of corresponding bulk pore-fluid molecules. In the case of liquids, the times are much shorter. Under appropriate circumstances of rapid exchange, average rates may be observed [4]. Careful analysis of the relaxation time distribution can usually lead to an estimate of the pore surface to volume ratio. In more complex experiments, the connectivity of pores and the exchange rate of molecules between them can be estimated.

Notwithstanding all the progress that has been made, it can be difficult to definitively ascribe a model of relaxation coupled to a model of morphology to any given system. By and large, bulk fluids are well understood, but the relaxation of fluids in nanoscale geometries, and especially geometries of low dimensionality, are much less well understood. It is the purpose of this paper to build on previous work in three dimensions (3D) with a combination of analytical analyses, molecular dynamics (MD), and Monte Carlo (MC) simulations that lay a framework for efficient computation of relaxation in twodimensional (2D) and quasi-two-dimensional (Q2D) spaces. Among other systems, such nanoscale spaces are thought to be dominant in calcium-silicate-hydrate gels in cements, as shown schematically in Fig. 1. This work looks specifically at the relaxation of the porefluid in the 2D and Q2D spaces. Relaxation in pores is often dominated by surface interactions. Korb and co-workers [5] have suggested that 2D diffusion across pore surfaces is a critical relaxation mechanism. Molecular rotations are as important as translational motions in determining relaxation in bulk fluids [6]. In restricted geometries, this is not necessarily the case. MD simulations offer a pointer as to the relative importance of the two. In a followup paper, we will use the results learned here and add in interaction with, and diffusion across, the $2 \mathrm{D}$ pore surfaces to build a complete picture of the relaxation process. 


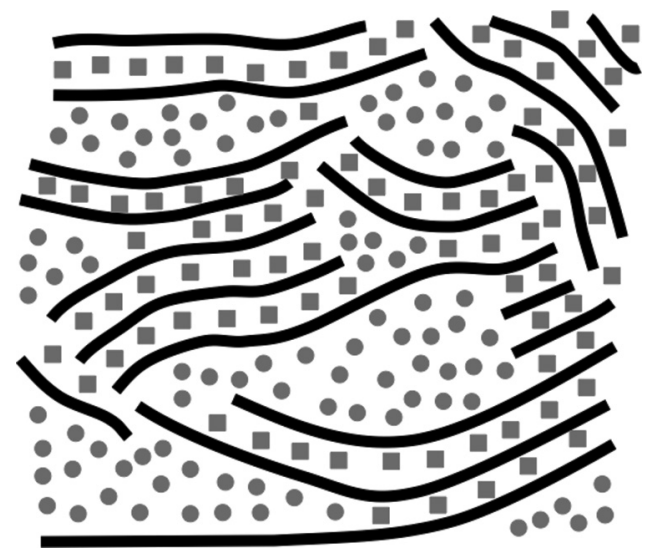

FIG. 1. A schematic view of the calcium-silicate-hydrate gel as proposed for cements. The gel is believed to comprise layers of calcium and oxygen atoms and silicate tetrahedra (solid lines) separated by sheets of water (filled square). Slightly larger gel pores circa 3-5 nm wide and filled with water (filled circle) separate regions of locally aggregated layers (from Ref. [1]).

Analytic models by Korb and co-workers [5,7-9], Kimmich and co-workers [10,11], and Levitz and co-workers [12-14], among others, which incorporate generalized surface interactions and diffusion processes, have been shown to provide good fits to experimental data in the low NMR frequency regime. Numerical modeling can access higher frequencies and can potentially provide a more accurate picture of the nanoscale dynamics in specific morphologies, not least in calcium-silicate hydrates.

The relaxation times $T_{1}$ and $T_{2}$ are simple functions of a spectral density function $J(\omega)$, which describes the frequency dependence of intermolecular magnetic interactions and which, in turn, is the time Fourier transform of the timedependent magnetic dipole-dipole correlation function [6]. This correlation function which, following common usage, we refer to as $G^{*}(t)$ in this paper, contains information about the relative motion of pairs of spins. Hence, it is the determination of the correlation function $G^{*}(t)$ that is necessary for the calculation of the relaxation times $T_{1}$ and $T_{2}$. If the local nanostructure modifies the dynamics, this is reflected in $G^{*}(t)$ and hence the relaxation times are modified compared to the bulk fluid.

The theoretical calculation of $G^{*}(t)$ requires a model for the dynamics of magnetic spins for the system of interest. $G^{*}(t)$ depends on the relative motion of pairs of spins governed by the conditional probability density function $p\left(\mathbf{r}, t \mid \mathbf{r}_{0}\right)$, which is the probability that a pair of spins is separated by $\mathbf{r}$ at time $t$ and by $\mathbf{r}_{0}$ at time $t=0$. Any attempt at calculating the correlation function must access the probability density function either by determining an analytic expression for $p\left(\mathbf{r}, t \mid \mathbf{r}_{0}\right)$ or by executing a numerical simulation for an ensemble of spins.

The simplest model system comprises a fluid of noninteracting spins. If each spin is assumed to move independently and unhindered in a bulk fluid characterized only by the selfdiffusion coefficient, $p\left(\mathbf{r}, t \mid \mathbf{r}_{0}\right)$ can be obtained by a routine solution to the diffusion equation for a delta-function source. This is the basis of the Bloembergen, Purcell, and Pound (BPP) model [15] which was developed by Torrey [16] and Abragam and is presented in Abragam's classic text [6]. This fluid is characterized by a uniform spin radial density function beyond a distance $\delta$, which can be considered as the distance of nearest approach. We refer to this fluid as a "uniform" fluid in this paper. The theory yields relatively simple expressions for the correlation function, the spectral density function, and hence the relaxation times. The uniform fluid solution remains useful for comparison with more complex diffusion models and as a good representation of most diffusing spin systems in the long-time limit. Also, as explained in the following section, it can help overcome some of the shortcomings of numerical simulations for the determination of $G^{*}(t)$.

For real molecules, the assumption of a noninteracting system is not appropriate and $p\left(\mathbf{r}, t \mid \mathbf{r}_{0}\right)$ is difficult to determine over the full time domain even for simple diffusion models. A Monte Carlo simulation was developed by Sholl and coworkers [17] for the site-blocking, simple-hopping model in lattice systems because the correlated motion of spins at short time scales was inaccessible to a theoretical interpretation. This work, which was motivated by the study of the diffusion of hydrogen in metals, used computer simulation methods directly to calculate NMR relaxation times.

The focus of this paper is the study of hydrated systems with reduced dimensionality, specifically 2D and Q2D systems in which the water is confined to thicknesses of a few nanometers in the $z$ direction by planar surfaces. Of special relevance to the present studies is the theoretical work of Korb and co-workers [5,7-9], who separate surface diffusion from bulk diffusion in Q2D pores providing a good fit to experimental data of the NMR relaxation time $T_{1}$ as a function of frequency in protonated porous glass, cement, and protein systems. According to this model, molecules diffusing on a surface have an enhanced probability of repeat encounters with adsorbed relaxation centers. Experimental results on cement paste in particular suggest that water primarily inhabits a network of planar $(1 \mathrm{~nm})$ interlayer spaces linked to larger Q2D pores known as gel pores (3-5 nm) [18]. Water appears to diffuse by different mechanisms over time scales from nanoseconds to milliseconds. NMR relaxation measurements on cement pastes, interpreted using the Korb model [5,18], suggest a water correlation time for pore-surface diffusion events of the order of nanoseconds, a pore surface residency time of the order of microseconds and a pore-pore exchange time of the order of milliseconds. The last result is puzzling because the diffusion coefficient of water, of the order of $10^{-9} \mathrm{~m}^{2} / \mathrm{s}$, suggests that a water molecule moves a distance of the order of microns during a time period of $1 \mathrm{~ms}$, at odds with common models of the nanostructure of cement. These results serve to emphasize the need for numerical simulation to identify key mechanisms for the diffusion of spins in order to aid the interpretation of NMR data.

The models of Korb and co-workers [5,8,9] are concerned with the calculation of relaxation rates due to the movement of water relative to stationary surface paramagnetic impurities. Surface paramagnetic impurities are known to dominate the relaxation in many systems in the "fast exchange" limit [19]. The effect of paramagnetic impurities is not considered in this work, but this contribution can be calculated from the simulations without the need for additional spin-trajectory calculations. 
Previous work on surface-mediated diffusion also includes that of Kimmich and co-workers [10,11] who explored the diffusion dynamics of surface random walkers in the strong-adsorption regime. Here, adsorbed spins are subject to occasional bulk relaxation steps. The diffusion probability density function departs from the normal Gaussian behavior and exhibits Lévy statistics. Computed correlation functions were compared to MC simulation, leading to the conclusion that the correlation function is dominated by Cauchy statistics for timescales less than the surface retention time. Levitz and co-workers [12] studied one-dimensional motion of spins diffusing along a rigid filament interspersed by bulk relocations. NMR experiments of the water spin-lattice relaxation rate were undertaken in colloidal suspensions supporting the basis of the diffusion model. Later work by Levitz and co-workers explored the behavior of adsorbates in spherical pores with the surface diffusion and bulk diffusion characterized by different diffusion coefficients [13,14].

The limitation of previous studies is that the theoretical analysis is primarily limited to a restricted time scale, usually to long times, and hence to low frequencies. Numerically efficient methods of calculating $G^{*}(t)$ for systems of lower dimensionality characteristic of a range of materials are not known and it is not possible to distinguish the relative importance of competing effects contributing to measured relaxation times. This work explores the behavior of water in 2D and Q2D systems using simple-hopping, site-blocking Monte Carlo (MC) simulation. Molecular dynamics (MD) simulations of bulk water and water in Q2D systems are undertaken for comparison and to parametrize the MC simulations. MD accesses length scales of nanometers over a time scale of nanoseconds whereas MC simulations extend to microseconds. The correlation function $G^{*}(t)$ is determined directly from the simulations.

A number of workers have undertaken MC and MD simulations for the purpose of NMR relaxation times since the first lattice-based MC simulations in 1986 [17]. For example, Hua and co-workers studied a disordered system using MC targeting metal hydrides [20] and Mattea and co-workers [21] used MC simulation to determine the spectral density function for diffusion in nanoscopic spaces with hydrodynamic flow. Bergenstråhle and co-workers [22] used atomistic simulation of cellulose-water interfaces to calculate $T_{1}$ and Grivet [23] undertook a study of a Lennard-Jones fluid using MD and discusses the limitations of MD simulation for extracting relaxation times for water. However, just as theoretical analyses are limited to (usually) long-time limits amenable to stochastic averaging, so numerical simulations are constrained by computational resources to limited simulation cell size and simulation time.

The specific contributions made in this paper are as follows. First, we show that numerical simulation and analytical theory complement each other to yield the correlation function over the full time domain. An analytical correction term is presented for addition to the correlation function obtained from the $2 \mathrm{D}$ $\mathrm{MD}$ and MC simulations to account for the contribution of spin pairs beyond those accessible in the numerical model. The derivation of the $2 \mathrm{D}$ term based on the work of Abragam and others in 3D [6,15-17] is given in Appendix B and presented as Eq. (20). Second, we provide an explicit expression for the correlation function for a uniform 2D fluid $G_{\mathrm{u}, 2 \mathrm{D}}^{*}(t)$. A uniform fluid is a fluid in which a spin is surrounded by a uniform density of spins beyond a distance $\delta$ which can be considered as the distance of nearest approach of the spins. This follows from Eq. (20) and serves as a useful reference system for comparison to the simulations. Third, we show that the 3D Sholl powderaveraging formula [24] for the correlation function applies equally to 2D and Q2D systems. This result provides a simple and computationally efficient technique to use in numerical simulations. Fourth, we introduce an analytical expression for the $2 \mathrm{D}$ correlation function applicable in the long-time limit. It is well known that $G^{*}(t) \rightarrow A t^{-3 / 2}$ for $3 \mathrm{D}$ systems and $G^{*}(t) \rightarrow B t^{-1}$ for $2 \mathrm{D}$ and Q2D systems. We determine the constant $B$. Finally, we demonstrate that the $T_{1}$ associated with both the translational and rotational motion of spins decreases as the pore thickness is reduced.

The paper is organized as follows. The theoretical framework for the interpretation of the numerical simulations is presented in Sec. II. Details of the numerical simulations are presented in Sec. III and the results of the MD and MC simulations are presented in Sec. IV. Finally, the results are summarized and conclusions drawn in Sec. V.

\section{THEORY}

\section{A. General background}

A collection of spins associated with the same nuclear species diffuses in a volume of space in the presence of a static magnetic field. The dipole-dipole interaction of pairs of spins is modified by their relative translational diffusion and rotational motion. The spin-lattice $\left(T_{1}\right)$ and spin-spin $\left(T_{2}\right)$ relaxation times are expressed in terms of spectral density functions $J^{(m)}(\omega)$ by the well-known equations [6]

$\frac{1}{T_{1}}=\frac{3}{2}\left(\frac{\mu_{0}}{4 \pi}\right)^{2} \gamma^{4} \hbar^{2} I(I+1)\left[J^{(1)}(\omega)+J^{(2)}(2 \omega)\right]$,

$\frac{1}{T_{2}}=\frac{3}{8}\left(\frac{\mu_{0}}{4 \pi}\right)^{2} \gamma^{4} \hbar^{2} I(I+1)\left[J^{(0)}(0)+10 J^{(1)}(\omega)+J^{(2)}(2 \omega)\right]$,

where $\gamma$ and $I$ are the gyromagnetic ratio and spin of the diffusing spins and $\omega$ is the Larmor frequency in the applied static field. The spectral density functions $J^{(m)}(\omega)$ are obtained from the Fourier transformation of the associated correlation functions $G^{(m)}(t)$ :

$$
\begin{gathered}
J^{(m)}(\omega)=\int_{-\infty}^{\infty} G^{(m)}(t) e^{-i \omega t} d t, \\
G^{(m)}(t)=\alpha^{(m)}\left\langle\frac{Y_{2 m}\left(\theta_{0}, \phi_{0}\right) Y_{2 m}^{*}(\theta, \phi)}{r_{0}^{3} r^{3}}\right\rangle,
\end{gathered}
$$

where

$$
\alpha^{(0)}=\frac{48 \pi}{15}, \quad \alpha^{(1)}=\frac{8 \pi}{15}, \quad \alpha^{(2)}=\frac{32 \pi}{15} .
$$

Spin-pair vectors are $\mathbf{r}_{0}$ and $\mathbf{r}$ at $t=0$ and at time $t$, respectively, with spherical polar coordinates $\left(r_{0}, \theta_{0}, \phi_{0}\right)$ and $(r, \theta, \phi)$, respectively, in the laboratory frame of reference (frame $C$ ) in which the polar axis is in the direction of the static magnetic field. We use the usual notation that a vector quantity is indicated by a bold symbol and the magnitude of the vector 
by the normal symbol. Thus, $r=|\mathbf{r}|$. The $Y_{2 m}(\theta, \phi)$ are the spherical harmonic functions and the superscript asterisk on the spherical harmonic indicates the complex conjugate. The ensemble average represented by Eq. (3) is the average over all spin pairs. The ensemble is characterized by a probability density function $p\left(\mathbf{r}, t \mid \mathbf{r}_{0}\right)$, often referred to as the diffusion propagator, where $p\left(\mathbf{r}, t \mid \mathbf{r}_{0}\right)$ is the probability that a pair of spins is separated by $\mathbf{r}_{0}$ at time $t=0$ and by $\mathbf{r}$ at time $t$.

There are two approaches to the determination of $G^{(m)}(t)$ using Eq. (3). One approach is to assume a diffusion model and to determine an analytic expression for $G^{(m)}(t)$, for example, by solving the diffusion equation. Alternatively, the correlation functions may be determined by sampling the probability density function $p\left(\mathbf{r}, t \mid \mathbf{r}_{0}\right)$ via a numerical simulation.

A numerical simulation generates spin ensembles and the ensemble average represented by Eq. (3) can be expressed as

$$
G^{(m)}(t)=\frac{\alpha^{(m)}}{N} \sum_{r_{0}, r} \frac{Y_{2 m}\left(\theta_{0}, \phi_{0}\right) Y_{2 m}^{*}(\theta, \phi)}{r_{0}^{3} r^{3}}
$$

for $N$ spins where a spin is excluded from interacting with itself. It is convenient to introduce the correlation function $G^{*}(t)$ defined as

$$
G^{*}(t)=\frac{4 \pi G^{(m)}(t)}{\alpha^{(m)}}=\frac{4 \pi}{N} \sum_{r_{0}, r} \frac{Y_{2 m}\left(\theta_{0}, \phi_{0}\right) Y_{2 m}^{*}(\theta, \phi)}{r_{0}^{3} r^{3}},
$$

which is consistent with the notation used by Sholl [17] and, as will be demonstrated later, is independent of $m$.

The summation appearing in Eqs. (4) and (5) indicates that the correlation function is determined by summing the contribution of $N-1$ spin pairs for each of $N$ spins. In this work, the average is determined directly from MD and MC simulations where the system size is limited by computational constraints. In practice, therefore, the average is executed for all spin pairs located within a distance $d$ of each spin at $t=0$. The cutoff distance $d$ is one half the dimension of the simulation box. The total correlation function $G^{*}(t)$ can therefore be divided into four contributory components $[17,23]$

$$
G^{*}(t)=G_{\mathrm{NN}}^{*}(t)+G_{\mathrm{NF}}^{*}(t)+G_{\mathrm{FN}}^{*}(t)+G_{\mathrm{FF}}^{*}(t),
$$

where the subscripts refer, respectively, to the "near-near" spin contributions such that $r_{0} \leqslant d, r \leqslant d$, "near-far" such that $r_{0} \leqslant$ $d, r>d$, "far-near" where $r_{0}>d, r \leqslant d$, and "far-far" where $r_{0}>d, r>d$. Analytic approximations for all contributions except $G_{\mathrm{NN}}^{*}(t)$ were derived for early 3D MC simulations [17]. MD or MC simulations are used to determine all contributions except for $G_{\mathrm{FF}}^{*}(t)$ assuming that $G_{\mathrm{FN}}^{*}(t)=G_{\mathrm{NF}}^{*}(t)$. Expressions for $G_{\mathrm{FF}}^{*}(t)$ are presented in Sec. II C.

\section{B. Powder average}

Samples normally contain powdered material with pores randomly orientated with respect to the magnetic field. The measured magnetization includes contributions from pores with an assumed uniform distribution of orientation with respect to the static magnetic field. The experiment measures the time dependence of the magnetization averaged over all geometries. This is generally assumed to be an exponential decay. The Laplace transform of the magnetization yields a narrow distribution of relaxation rates with an average relaxation rate $\left\langle T_{1}^{-1}\right\rangle$ or $\left\langle T_{2}^{-1}\right\rangle$, characteristic of the average molecular environment.

Simulations and analytic models yield the relaxation rate for a pore with a specific orientation with respect to the magnetic field. The "powder-average" relaxation rate is achieved by taking the average of the relaxation rate over all orientations of the pore or, equivalently, over all magnetic field directions. The experimentally equivalent process requires separate measurement of the magnetization decay for each pore. The magnetization would be Laplace transformed to yield the porespecific relaxation rate and the average relaxation rate could thereafter be calculated. Hence, simulations and analytical models differ from experiment insomuch as models take the average over magnetic field directions while experiments take the average of the magnetization, as noted by Wolf $[25,26]$. This approximation is acceptably accurate [5,27]. For example, Korb et al. demonstrate that determining $\left\langle T_{1}^{-1}\right\rangle$ or $\left\langle T_{2}^{-1}\right\rangle$ by taking the average over magnetic field directions rather than the average of the magnetization introduces an error of no more than about $3 \%$ [5].

The following sections describe the derivation of a formula which enables the numerically efficient computation of the powder-average correlation function for $3 \mathrm{D}, 2 \mathrm{D}$, and $\mathrm{Q} 2 \mathrm{D}$ systems from numerical simulation.

\section{1. $3 D$ systems}

The powder-average correlation function for the translational diffusion of spins in $3 \mathrm{D}$ is derived by taking the average over all orientations of the system with respect to a fixed laboratory frame of reference $C$ in which the $z$ direction is aligned along the direction of the static magnetic field. A second frame of reference is the $C^{\prime}$ frame. We use the notation that a prime superscript indicates a quantity in the $C^{\prime}$ frame, a quantity in the $C$ frame is unprimed, and the subscript " 0 " is shorthand for $t=0$. For example, $\mathbf{r}_{0}$ has coordinates $\left(x_{0}, y_{0}, z_{0}\right) \equiv\left(r_{0}, \theta_{0}, \phi_{0}\right)$ in the $C$ frame and coordinates $\left(x_{0}^{\prime}, y_{0}^{\prime}, z_{0}^{\prime}\right) \equiv\left(r_{0}, \theta_{0}^{\prime}, \phi_{0}^{\prime}\right)$ measured in the $C^{\prime}$ frame, noting of course that $r_{0}=r_{0}^{\prime}$.

In order to determine the powder-average correlation function, it is necessary to express the spherical harmonic function $Y_{2 m}(\theta, \phi)$ for a given spin pair in the laboratory frame $C$ in terms of its spherical harmonic function $Y_{2 m}\left(\theta^{\prime}, \phi^{\prime}\right)$ determined with respect to the frame $C^{\prime}$. The spherical harmonic function $Y_{2 m}(\theta, \phi)$ can be calculated using the transformation [24,28]

$$
Y_{2 m}(\theta, \phi)=\sum_{M=-2}^{2} d_{m M} Y_{2 M}\left(\theta^{\prime}, \phi^{\prime}\right) .
$$

The expansion coefficients $d_{m M}$ may be determined efficiently using the method of Romanowski and Krukowski [29] as summarized in Appendix A. In this method, the coordinate frame $C^{\prime}$ is obtained by rotating the frame $C$ anticlockwise about the vector $\mathbf{u}$ (as viewed looking along $\mathbf{u}$ from its positive end towards the origin) through an angle $\alpha$. In the present case, powder averaging may be achieved by choosing the $C^{\prime}$ frame such that $z^{\prime}$ is orientated along $\mathbf{r}_{0}$. The axis of rotation is therefore perpendicular to both the $z$ axis and to $\mathbf{r}_{0}$. The unit vector $\mathbf{u}$ is obtained by taking the vector product of $\hat{\mathbf{k}}=(0,0,1)$ with $\mathbf{r}_{0}$ so that $\mathbf{u}=\left(-\sin \phi_{0}, \cos \phi_{0}, 0\right)$. Since the angle of 
rotation $\alpha=\theta_{0}$, where $\theta_{0}$ is the polar angle of the vector $\mathbf{r}_{0}$ as measured in the $C$ frame, it is evident that the expansion coefficients $d_{m M}$ in Eq. (7) are functions of the angles $\left(\theta_{0}, \phi_{0}\right)$.

Equation (7) is substituted into Eqs. (4) and (5) to yield

$$
G^{*}(t)=\frac{4 \pi}{N} \sum_{r_{0}, r} \frac{Y_{2 m}\left(\theta_{0}, \phi_{0}\right)}{r_{0}^{3} r^{3}} \sum_{M=-2}^{2} d_{m M}^{*} Y_{2 M}^{*}\left(\theta^{\prime}, \phi^{\prime}\right) .
$$

The powder average is then achieved by, first, averaging over the azimuthal angle $\phi^{\prime}$ and then over the polar angles of $\mathbf{r}_{0}$, namely, $\phi_{0}$ and $\theta_{0}$, thus

$$
\begin{aligned}
G^{*}(t)= & \frac{4 \pi}{N} \sum_{r_{0}, r} \frac{1}{r_{0}^{3} r^{3}} \sum_{M=-2}^{2} \frac{1}{4 \pi} \\
& \times\left[\int_{0}^{2 \pi} \int_{0}^{\pi} d_{m M}^{*}\left(\theta_{0}, \phi_{0}\right) Y_{2 m}\left(\theta_{0}, \phi_{0}\right) \sin \theta_{0} d \theta_{0} d \phi_{0}\right] \\
& \times\left[\frac{1}{2 \pi} \int_{0}^{2 \pi} Y_{2 M}^{*}\left(\theta^{\prime}, \phi^{\prime}\right) d \phi^{\prime}\right]
\end{aligned}
$$

where the formal dependence of the expansion coefficients on the angles $\left(\theta_{0}, \phi_{0}\right)$ is recognized. By inspection, when the integral over $\phi^{\prime}$ is taken, all terms in which the spherical harmonic $Y_{2 M}^{*}\left(\theta^{\prime}, \phi^{\prime}\right)$ contains the angle $\phi^{\prime}$ vanish. This leaves the $M=0$ term only for each $m$. The integral over $\phi_{0}$ then leaves a single nonzero term corresponding to $m=0, M=0$. The final integral over $\theta_{0}$ yields the well-known expression

$$
\begin{aligned}
G^{*}(t) & =\frac{1}{N} \sum_{r_{0}, r} \frac{\frac{1}{2}\left(3 \cos ^{2} \psi-1\right)}{r_{0}^{3} r^{3}} \\
& =\left\langle\frac{P_{2}(\cos \psi)}{r_{0}^{3} r^{3}}\right\rangle,
\end{aligned}
$$

where we have used the symbol $\psi$ to represent the angle between $\mathbf{r}$ and $\mathbf{r}_{\mathbf{0}}$ (so that $\left.\psi=\theta^{\prime}\right) . P_{2}(x)=\frac{1}{2}\left(3 \cos ^{2} x-1\right)$ is a Legendre polynomial. The angular bracket on the right-hand side of Eq. (11) represents the average over an ensemble of spin pairs. This constitutes a numerically efficient method of determining the powder-average correlation function from a numerical simulation. The same result was derived by a slightly different approach by Sholl [24] based on the work of Messiah [28].

\section{2. $2 D$ systems}

For $2 \mathrm{D}$ systems, the $C^{\prime}$ frame is chosen such that the $z^{\prime}$ axis is normal to the plane of the 2D diffusion as illustrated in Fig. 2. Thus, $\theta^{\prime}=\theta_{0}^{\prime}=\pi / 2$. The spherical harmonic functions $Y_{2 m}(\theta, \phi)$ required for Eq. (4) can be determined using the transformation given by Eq. (7). There is an equivalent expression for $Y_{2 m}\left(\theta_{0}, \phi_{0}\right)$ with expansion coefficients denoted $d_{m M_{0}}$. The $d_{m M}$ are functions of the angle(s) of rotation and, as previously, may be determined using the method of Romanowski and Krukowski [29]. Substitution of Eq. (7) into (4) yields a double summation involving the product of rotation coefficients $d_{m M_{0}} d_{m M}^{*}$. The powder average may be achieved by applying a sequence of just two rotations through angles $\alpha$ and $\beta$, provided one rotation is in plane, and then taking the angular average over each. Rotations about the $z$ and $y$ axes (see Fig. 2) suffice. The average over the angles $\alpha$

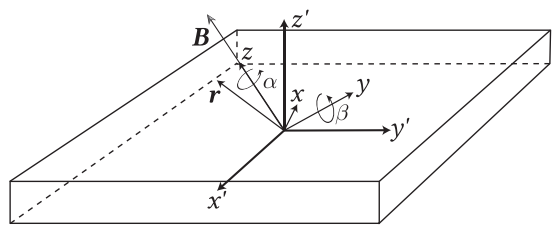

FIG. 2. The spin-pair vector $\mathbf{r}$ has coordinates $(x, y, z) \equiv(r, \theta, \phi)$ in the $C$ (laboratory) frame of reference and coordinates $\left(x^{\prime}, y^{\prime}, z^{\prime}\right) \equiv$ $\left(r, \theta^{\prime}, \phi^{\prime}\right)$ in the $C^{\prime}$ (simulation) frame. The magnetic field acts in the $z$ direction. The frame $C^{\prime}$ is obtained by rotating the frame $C$ anticlockwise about the $x$ axis through angle $\alpha$ followed by a rotation about the $y$ axis through angle $\beta$.

and $\beta$ is then obtained from

$$
\left\langle d_{m M_{0}} d_{m M}^{*}\right\rangle_{\alpha \beta}=\frac{1}{4 \pi} \int_{0}^{2 \pi} \int_{0}^{\pi} d_{m M_{0}} d_{m M}^{*} \sin \beta d \beta d \alpha .
$$

All integrals vanish except for $M_{0}=M$ where the result is $\frac{1}{5}$ for each of $m=0,1$, and 2. This is in accordance with the result of Sholl [24]. The correlation function in 2D is now given by

$G_{2 \mathrm{D}}^{*}(t)=\frac{4 \pi}{5 N} \sum_{r_{0}, r} \frac{1}{r_{0}^{3} r^{3}}\left[\sum_{M= \pm 2,0} Y_{2 M}\left(\frac{\pi}{2}, \phi_{0}^{\prime}\right) Y_{2 M}^{*}\left(\frac{\pi}{2}, \phi^{\prime}\right)\right]$,

where it is noted that the $M=1$ terms in the summation vanish. As previously, we now define $\psi$ as the angle between $\mathbf{r}$ and $\mathbf{r}_{0}$. In $2 \mathrm{D}, \psi=\phi-\phi_{0}$. Making this substitution into Eq. (13) yields the result

$$
\begin{aligned}
G_{2 \mathrm{D}}^{*}(t) & =\frac{1}{N} \sum_{r_{0}, r} \frac{\frac{1}{2}\left(3 \cos ^{2} \psi-1\right)}{r_{0}^{3} r^{3}} \\
& =\left\langle\frac{P_{2}(\cos \psi)}{r_{0}^{3} r^{3}}\right\rangle,
\end{aligned}
$$

which is identical to Eq. (11). In other words, the same calculation is required in order to determine the powderaveraged correlation function from the numerical simulation in both 2D and 3D.

\section{Q2D systems}

The derivation of identical expressions given by Eqs. (11) and (15) for the determination of the powder-average correlation function for spins diffusing in $3 \mathrm{D}$ and $2 \mathrm{D}$, respectively, despite the different theoretical approaches, suggests that these expressions are of general validity. The averaging procedure used for the 3D system is a mathematically efficient method of obtaining the powder average because the single angle of rotation required to obtain $C^{\prime}$ from $C$ is the angle $\psi$. This mathematical approach is equally applicable to all systems regardless of dimensionality. This deduction that expression (11) is generally applicable was checked by the numerical computation of $G^{*}(t)$, first using Eq. (11) directly and, second, taking a numerical average over a sample of magnetic field directions for $2 \mathrm{D}, 3 \mathrm{D}$, and $\mathrm{Q} 2 \mathrm{D}$ systems. The numerical results confirm that Eq. (15) is of general validity. The applicability of Eq. (15) to 2D and Q2D systems for the calculation of the powder-average correlation function is 
critical to the efficient calculation of $G^{*}(t)$ in low-dimensional systems but has not been appreciated before.

\section{Determination of $G_{\mathrm{FF}}^{*}(t)$}

This section describes the determination of the "far-far" correction to the correlation function $G^{*}(t)$. The correction $G_{\mathrm{FF}}^{*}(t)$ is added to $G^{*}(t)$ determined from the simulation [Eq. (6)] to approximately account for pairs of spins for which both $r_{0}>d$ and $r>d$, where $d$ is half the simulation cell dimension. $G_{\mathrm{FF}}^{*}(t)$ is negligible at short times for the simulation box sizes used in the current work in which $2 d \gtrsim 0.4 \mathrm{~nm}$. However, it provides an increasingly significant contribution to the total correlation function as time increases.

The derivation of $G_{\mathrm{FF}}^{*}(t)$ for both $3 \mathrm{D}$ and 2D systems relies on an appropriate analytic expression for the diffusion propagator $p\left(\mathbf{r}, t \mid \mathbf{r}_{0}\right)$, the probability that a pair of spins is separated by $\mathbf{r}$ at time $t$ and by $\mathbf{r}_{0}$ at $t=0$, where

$$
p\left(\mathbf{r}, t \mid \mathbf{r}_{0}\right)=P\left(\mathbf{r}, t \mid \mathbf{r}_{0}\right) p\left(\mathbf{r}_{0}\right) .
$$

$P\left(\mathbf{r}, t \mid \mathbf{r}_{0}\right)$ is the probability that a pair of spins is separated by $\mathbf{r}$ at time $t$ given that the pair was separated by $\mathbf{r}_{0}$ at $t=0$ and $p\left(\mathbf{r}_{0}\right)$ is the a priori probability that a spin-pair vector can take the value $\mathbf{r}_{0}$. The probability $P\left(\mathbf{r}, t \mid \mathbf{r}_{0}\right)$ is determined for uncorrelated spins as a standard solution to the diffusion equation for a delta-function source and may be written in $n$ dimensions as

$$
P\left(\mathbf{r}, t \mid \mathbf{r}_{0}\right)=\prod_{i=1, n} \frac{e^{-x_{i}^{2} / 8 D t}}{(8 \pi D t)^{1 / 2}},
$$

noting that the relative displacement of a pair of uncorrelated spins at time $t$ is the same as if one spin is fixed at the origin and the other moving for a time $2 t$.

\section{1. $3 D$ systems}

In 3D, an expression for $G_{\mathrm{NF}}^{*}(t)+G_{\mathrm{FN}}^{*}(t)+G_{\mathrm{FF}}^{*}(t)$ was derived by Faux et al. [17] based on the model presented by Abragam [6]. The model assumes the diffusion propagator given by Eq. (17) for $n=3$. The specific contribution $G_{\mathrm{FF}}^{*}(t)$ may be expressed as

$$
G_{\mathrm{FF}, 3 \mathrm{D}}^{*}(t)=4 \pi N_{v} \int_{0}^{\infty} e^{-2 D t k^{2}} \frac{J_{3 / 2}^{2}(k d)}{k d^{3}} d k,
$$

where $J_{3 / 2}(x)$ is a half-integer Bessel function and $D$ is the self-diffusion coefficient. $N_{v}$ is the spin density (number of spins per unit volume) and $k$ is the Fourier variable which appears when the diffusion propagator given by Eq. (17) is expressed as a Fourier integral [6]. Equation (18) was not explicitly presented in Ref. [17]. Although an analytic solution of this integral is possible, direct numerical integration is straightforward and easier to compute at short times.

It is instructive to determine the limiting value of $G_{\mathrm{FF}, 3 \mathrm{D}}^{*}(t)$ as $t \rightarrow 0$. Placing the exponential in Eq. (18) equal to unity and expanding the Bessel function leads to the result

$$
G_{\mathrm{FF}, 3 \mathrm{D}}^{*}(0)=\frac{4 \pi N_{v}}{3 d^{3}} .
$$

Note that we believe that there is a typographical error in Eq. (3.5) of [17].

\section{2. $2 D$ systems}

A similar method for the derivation of the equivalent correction term for $2 \mathrm{D}$ translational diffusion $G_{\mathrm{FF}, 2 \mathrm{D}}^{*}(t)$ is presented in Appendix B. The final result is

$$
\begin{aligned}
G_{\mathrm{FF}, 2 \mathrm{D}}^{*}(t)= & \frac{4 \pi N_{s}}{8} \int_{0}^{\infty} k e^{-2 D t k^{2}}\left(3\left[\int_{d}^{\infty} \frac{J_{2}(k r)}{r^{2}} d r\right]^{2}\right. \\
& \left.+\left[\int_{d}^{\infty} \frac{J_{0}(k r)}{r^{2}} d r\right]^{2}\right) d k
\end{aligned}
$$

where $N_{s}$ is the areal spin density. This expression does not usefully simplify further and the integrals must be computed numerically.

\section{D. $G^{*}(t)$ for a uniform fluid}

The expressions for the "far-far" correction terms presented in Eqs. (18) and (20) can be readily adapted to produce an expression for $G^{*}(t)$ for a 3D or 2D uniform fluid over the full time domain. We identify this function as $G_{\mathrm{u}}^{*}(t)$. A uniform fluid in this context is one with a radial density function $g(r)$ of the following form:

$$
\begin{aligned}
g(r) & =0(r<\delta) \\
& =1(r \geqslant \delta) .
\end{aligned}
$$

Thus, $G_{\mathrm{u}}^{*}(t)$ is obtained using Eqs. (18) and (20) by excluding spin pairs closer than the chosen cutoff distance $\delta$ and setting $d=\delta . \delta$ was considered by Abragam to be the distance of nearest approach of two spins [6], but the pair radial density function for a real fluid is not uniform and the correlation function obtained from a MD simulation reflects the nonuniform spin-pair density at short distances. Later, we will see that the cutoff distance $\delta$ can be chosen to yield the correct value of the correlation function at $t=0$ based on the MD results.

\section{E. Determination of $G^{*}(t \rightarrow \infty)$}

It is useful to determine the simple expressions for the powder-average correlation functions in the long-time limit. These may be evaluated for 3D, 2D, and Q2D systems and are of the form $A / t^{3 / 2}, B / t$, and $C / t$, respectively. The constants $A, B$, and $C$ are determined for diffusion on cubic or square lattices. These constitute approximations for the long-time behavior of $G^{*}(t)$ for direct comparison to the MD and MC numerical simulations.

\section{3D spin systems}

The mathematical treatment for 3D systems follows that of Sholl [30] and assumes that the spins execute random hops on a discrete lattice. Starting with Eq. (10) and rewriting the summation over all spin pairs by a double summation over all lattice vectors gives

$$
G_{3 \mathrm{D}}^{*}(t)=c \sum_{i} \frac{1}{r_{i}^{3}} \sum_{j} \frac{P_{2}\left(\cos \psi_{i j}\right)}{r_{j}^{3}} P\left(\mathbf{r}_{j}, t \mid \mathbf{r}_{i}\right) .
$$

Each summation is taken over the set of lattice sites. $\psi_{i j}$ is the angle between the lattice vectors labeled $i$ and $j$. The $a$ 
priori probability that a site is occupied $c$ appears by virtue of Eq. (16).

At $t=0$, it is obvious that $P\left(\mathbf{r}_{j}, t \mid \mathbf{r}_{i}\right)=1$ if $i=j$ and zero otherwise and, additionally, that $\psi_{i j}=0$. Equation (22) reduces to

$$
G_{3 \mathrm{D}}^{*}(0)=c \sum_{i} \frac{1}{r_{i}^{6}}=\frac{c}{b^{6}} \sum_{i} \frac{1}{\rho_{i}^{6}}=\frac{c S_{0}}{b^{6}},
$$

where the dimensionless lattice vectors are $\rho_{i}=r_{i} / b, b$ is the cubic lattice parameter, and $S_{0}$ is the lattice summation. This expression is used to parametrize the MC simulations based on $G_{3 \mathrm{D}}^{*}(0)$ determined directly from the 3D MD simulation.

To establish the long-time behavior of $G_{3 \mathrm{D}}^{*}(t)$, it is assumed that the diffusion takes place on a 3D cubic lattice which is occupied to a concentration $c$ of spins. The probability density function $P\left(\mathbf{r}_{j}, t \mid \mathbf{r}_{i}\right)$ is approximated by Eq. (17) with the exponential set to zero in the long-time limit. The expression (22) now becomes

$$
G_{3 \mathrm{D}}^{*}(t \rightarrow \infty)=\frac{c \Omega}{b^{6}(8 \pi D t)^{3 / 2}} \sum_{i} \frac{1}{\rho_{i}^{3}} \sum_{j} \frac{P_{2}\left(\cos \psi_{i j}\right)}{\rho_{j}^{3}},
$$

where, as previously, $r=b \rho$. The volume per lattice site $\Omega$ is required because $P\left(\mathbf{r}_{j}, t \mid \mathbf{r}_{i}\right)$ is a volume probability now evaluated at discrete lattice sites. $\Omega$ is equal to $b^{3}$ and so the expression for the powder-average correlation function in $3 \mathrm{D}$ becomes

$$
G_{3 \mathrm{D}}^{*}(t \rightarrow \infty)=\frac{N_{v} L_{3 \mathrm{D}}}{(8 \pi D t)^{3 / 2}},
$$

where $N_{v}$ is the number of spins per unit volume equal to $c / b^{3}$. The dimensionless double lattice summation has been written as $L_{3 \mathrm{D}}$ where

$$
L_{3 \mathrm{D}}=\sum_{i} \frac{1}{\rho_{i}^{3}} \sum_{j} \frac{P_{2}\left(\cos \psi_{i j}\right)}{\rho_{j}^{3}}
$$

and where the lattice summations are for a cubic lattice.

Unfortunately, this double summation is conditionally convergent which means that the numerical value obtained depends on the method of summation [30]. A direct double summation including all lattice vectors within a specified radius, or within a cube of a specified dimension, yields a result of zero. It has been shown [31], however, that the summation may be executed by, first, summing the lattice vectors $\rho_{j}$ in the $x-y$ plane only (so that $\rho_{z, j}=0$ ) for a particular $\rho_{i}$ (convergence is slow). The process is then repeated for different values of $\rho_{i}$. The full solution, however, is accessible to analytic treatment for a spin fluid and is given by [31] as

$$
L_{3 \mathrm{D}}=\left(\frac{4 \pi}{3}\right)^{2} \text {. }
$$

It appears that Eq. (27) is valid for the cubic lattices and for any fluid model $[27,30]$. Equations (27) and (26) can therefore be used to describe the long-time behavior of the correlation function for bulk water as represented in both the MD and MC simulations.

\section{2. $2 D$ spin systems}

The derivation of the long-time behavior of the correlation function for a $2 \mathrm{D}$ square lattice follows a similar process as for $3 \mathrm{D}$ and the final result is

$$
G_{2 \mathrm{D}}^{*}(t \rightarrow \infty)=\frac{N_{s} L_{2 \mathrm{D}}}{b^{2}(8 \pi D t)},
$$

where $N_{s}$ is the areal spin density (equal to $c / b^{2}$ ). The lattice summation taken over a square lattice is not conditionally convergent and can be computed directly. $L_{2 \mathrm{D}}$ is found to be 20.4016 .

\section{Q2D spin systems}

Q2D systems comprise $n$ planes of lattice sites with each of the $n$ planes consisting of a square lattice. Each plane is assumed to extend in the $x$ and $y$ directions. The probability density function obtained as a solution to the diffusion equation has been presented by Korb and co-workers [5] and may be expressed, in the long-time limit, as

$$
P_{\mathrm{Q} 2 \mathrm{D}}\left(\mathbf{r}, t \mid \mathbf{r}_{0}\right)=\frac{1}{h} \frac{e^{-r^{2} / 8 D t}}{(8 \pi D t)},
$$

where $r^{2}=x^{2}+y^{2}$. The slab thickness is denoted by $h$ so that $h=n b$. Equation (29) is valid at times sufficiently long for the spin density to be uniform in the $z$ direction, accounting for the $h^{-1}$ term.

The long-time behavior of the correlation function for a Q2D system will therefore correspond to a 2D system of diffusing spins. Following the previous method, it can be shown that

$$
G_{\mathrm{Q} 2 \mathrm{D}}^{*}(t \rightarrow \infty)=\frac{N_{v} L_{\mathrm{Q} 2 \mathrm{D}}}{b(8 \pi D t)},
$$

where

$$
L_{\mathrm{Q} 2 \mathrm{D}}=\frac{1}{n} \sum_{i} \frac{1}{\rho_{i}^{3}} \sum_{j} \frac{P_{2}\left(\cos \psi_{i j}\right)}{\rho_{j}^{3}} .
$$

This lattice summation is more difficult to calculate than for the $3 \mathrm{D}$ cubic or $2 \mathrm{D}$ square lattices in the previous sections. The method of calculation is presented in Appendix $\mathrm{C}$ which provides values of $L_{\mathrm{Q} 2 \mathrm{D}}$ for different slab thicknesses.

\section{NUMERICAL SIMULATION}

Numerical simulations were undertaken using both MD and MC techniques. The MD method was applied to 3D (bulk water) and Q2D systems and the MC approach applied to 3D, 2D, and Q2D systems. Details of each method are described below and a summary of the systems studied is provided in Table I.

\section{A. MD simulations}

MD involves setting up the Cartesian coordinates of individual atoms in the system, defining the interatomic potentials and letting the system evolve according to Newton's laws of motion. MD simulations were performed on bulk water and four Q2D systems. The Q2D systems consisted of water sandwiched between ( $\left.\begin{array}{lll}1 & 0 & 0\end{array}\right)$ surfaces of alpha-quartz $\left(\mathrm{SiO}_{2}\right)$ acting as interface planes. A snapshot of a Q2D system is provided in Fig. 3. The use of several $\mathrm{SiO}_{2}$ layers is necessary for decoupling the water-water interaction across the solid surface (i.e., the thickness should exceed the water-water potential cutoff) and to conveniently incorporate periodic 
TABLE I. List of numerical simulations with system sizes and simulation length.

\begin{tabular}{ccc}
\hline \hline & MD & MC \\
\hline $3 \mathrm{D}$ & $4.95 \times 4.95 \times 4.95 \mathrm{~nm}$ & $30 \times 30 \times 30$ \\
& 4062 water molecules & $d=3.59 \mathrm{~nm}$ \\
& $1 \mathrm{~ns}$ & $0.05 \mu \mathrm{s}$ \\
& & $60 \times 60$ \\
& & $d=7.18 \mathrm{~nm}$ \\
Q2D & & $0.05 \mu \mathrm{s}$ \\
& $5.5 \times 5.2 \times 1.0 \mathrm{~nm}$ & $30 \times 30 \times 3$ \\
& 886 water molecules & $h=0.7 \mathrm{~nm}$ \\
& $1 \mathrm{~ns}$ & $0.5 \mu \mathrm{s}$ \\
& $5.5 \times 5.2 \times 1.5 \mathrm{~nm}$ & $30 \times 30 \times 5$ \\
& 1367 water molecules & $h=1.2 \mathrm{~nm}$ \\
& $1 \mathrm{~ns}$ & $0.5 \mu \mathrm{s}$ \\
& $5.5 \times 5.2 \times 2.0 \mathrm{~nm}$ & $30 \times 30 \times 10$ \\
& 1797 water molecules & $h=2.4 \mathrm{~nm}$ \\
& $1 \mathrm{~ns}$ & $0.5 \mu \mathrm{s}$ \\
$5.5 \times 5.2 \times 5.0 \mathrm{~nm}$ & $30 \times 30 \times 25$ \\
4339 water molecules & $h=6.0 \mathrm{~nm}$ \\
& $1 \mathrm{~ns}$ & $0.5 \mu \mathrm{s}$ \\
\hline \hline
\end{tabular}

boundary conditions. The SPC/E potential [32] was chosen for water-water interactions because it produces a diffusion coefficient in good agreement with experiment and because it is relatively simple, so allowing longer simulations. The CLAYFF potential [33] was applied for $\mathrm{SiO}_{2}$ crystal and surface. Each surface oxygen atom is terminated with a hydrogen atom. The $\mathrm{SiO}_{2}$-water interaction was described using the Lorentz-Berthelot mixing rules [34]. The DLPOLY package was used for all simulations $[35,36]$ with periodic boundary conditions applied in each direction.

\section{3D bulk water}

A system of 4062 water molecules in a cubic box was pre-equilibrated to a temperature of $300 \mathrm{~K}$ and zero pressure using isothermal-isobaric MD simulations. The equilibrated simulation box measured $4.95 \mathrm{~nm} \times 4.95 \mathrm{~nm} \times 4.95 \mathrm{~nm}$

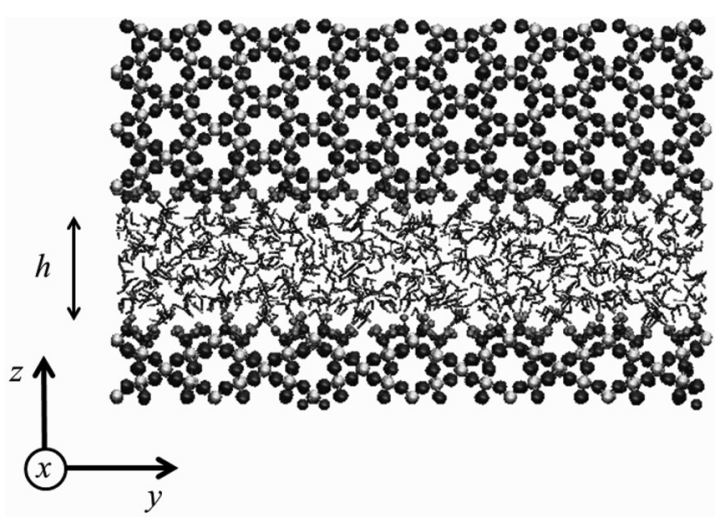

FIG. 3. A snapshot from MD simulations of Q2D water confined between (100) facets of alpha-quartz with $h=1.0 \mathrm{~nm}$. The atoms of the crystal are large spheres, surface hydroxyl are small spheres, and water molecules are shown as sticks. and was used to undertake a $1 \mathrm{~ns}$ simulation in the canonical ensemble. The simulation temperature was controlled using the Hoover thermostat [37]. The diffusion coefficient was calculated from the gradient of $\left\langle r^{2}\right\rangle$ as a function of time and found to be $2.7 \times 10^{-9} \mathrm{~m}^{2} / \mathrm{s}$, somewhat larger than the experimental diffusion coefficient of water at room temperature of $2.2 \times 10^{-9} \mathrm{~m}^{2} / \mathrm{s}$ [38]. However, Yeh and Hummer [39] have shown that the diffusion coefficient obtained in periodic simulations is system-size dependent. The value obtained here is in excellent agreement with Kerisit and Liu [40] for a cell dimension of about $4.95 \mathrm{~nm}$.

\section{Q2D simulations}

The system setup for the simulations of Q2D water confined between (100) facets of alpha quartz is illustrated in Fig. 3. The $\left(\begin{array}{lll}1 & 0 & 0\end{array}\right)$ surface was obtained by cleaving the bulk crystal at the corresponding crystallographic plane. The outermost oxygen sites on the quartz surface were hydroxylated to maintain the charge neutrality in the system. This is an idealized model surface which suits the purpose of our study. Note that the hydroxyl groups are not included in any calculation of the correlation function. $\mathrm{The}^{\mathrm{SiO}} \mathrm{S}_{2}$ slab measured $5.5 \mathrm{~nm} \times 5.2 \mathrm{~nm}$ in $x$ and $y$ directions and approximately $2.30 \mathrm{~nm}$ in the direction of the interface ( $z$ direction).

Four Q2D systems with pore water thicknesses $h$, approximately $1.0,1.5,2.0$, and $5.0 \mathrm{~nm}$ were simulated. The pores were filled with water to a density of approximately $1 \mathrm{~g} / \mathrm{cm}^{3}$ and equilibrated under isothermal-isobaric conditions to a target pressure of $0 \mathrm{~Pa}$. The final systems comprise 886, 1367, 1797, and 4339 water molecules, respectively. All Q2D simulations were then run for $1 \mathrm{~ns}$.

\section{B. MC simulations}

The MC simulations were executed using a simple-hopping, site-blocking diffusion model on a cubic lattice in 3D and Q2D and on a square lattice in 2D. Spins are placed at random to the desired concentration $c$ on the appropriate lattice and the diffusion process is modeled by choosing both a spin and the direction of an attempted hop at random. If the target site is vacant, the hop is accepted. If the target site is blocked by another spin or by a hard-wall boundary (in Q2D), the proposed hop is rejected and the process repeated. Periodic boundary conditions apply in the absence of a hard-wall boundary.

The choice of site occupancy fraction $c$ is dictated by the competing demands of the need for a large simulation cell (to avoid possible edge correlation effects and to reduce the contribution of the "far-far" correction to the correlation function), the need for a sufficiently large number of spins to secure adequate statistical averaging, and the need for sufficiently few spins to allow the simulation to extend to nearmicrosecond time scales. All MC simulations were undertaken with a fixed site occupancy fraction $c=0.228$ which was found to constitute a reasonable choice for all systems while balancing these competing demands.

\section{Parametrization of MC simulations}

Given a spin concentration $c$, the MC simulations are characterized by three parameters: a spin concentration scale 
TABLE II. The three MC parameters are determined using the equations and numerical values for the three listed physical observables. $f(c)$ is the tracer correlation factor given by Eq. (33) and $S_{0}$ is a lattice constant equal to 8.4019 (see text).

\begin{tabular}{lc}
\hline \hline MC parameters & Physical observable \\
\hline Lattice constant & Spin density \\
$b=0.239 \mathrm{~nm}$ & $N_{v}=66.6 \mathrm{spins} / \mathrm{nm}^{3}$ \\
Attempt jump time/spin & Self-diffusion coefficient \\
$\tau_{0}=3.13 \mathrm{ps}$ & $D=\frac{b^{2} f(c)(1-c)}{6 \tau_{0}}=0.0022 \mathrm{~nm}^{2} / \mathrm{ps}$ \\
Concentration scaling factor & Correlation function at $t=0$ \\
$s=4.0$ & $G_{\mathrm{MD}, 3 \mathrm{D}}^{*}(0)=\frac{s c S_{0}}{b^{6}}=40900 \mathrm{~nm}^{-6}$ \\
\hline \hline
\end{tabular}

factor $s$, the lattice spacing $b$, and the mean time between the attempted hops of a spin $\tau_{0}$. These parameters may be assigned by a number of methods, but we choose to parametrize so as to obtain the known spin density for bulk water at room temperature, the correct experimental self-diffusion coefficient, and to match $G_{3 \mathrm{D}, \mathrm{MD}}^{*}(0)$ as obtained from the MD simulation for bulk water.

The self-diffusion coefficient $D$ for particles at concentration $c$ diffusing on a lattice is given by

$$
D=\frac{b^{2} f(c)}{6 \tau},
$$

where $\tau$ is the mean time between actual jumps for a spin and is equal $\tau_{0} /(1-c)$ where $\tau_{0}$ is the mean time between attempted hops of a spin. In other words, for a system containing $N$ spins, a time $\tau_{0}$ has elapsed when a total of $N$ attempted hops have been made. $f(c)$ is the tracer correlation factor. It is less than unity for the site-blocking model and arises because a spin that has just executed a hop to a neighboring site has an enhanced probability of returning to its original site compared to the probability $1-c$ of moving to an alternative neighboring site.

An expression for $f(c)$ for the simple cubic lattice has been presented by Tahir-Kheli and Elliott [41]:

$$
f(c)=\left[1-\frac{2 c \theta}{(2-c)(1+\theta)}\right]^{-1},
$$

where $\theta=-0.2098$ for the simple cubic lattice.

The correlation function $G^{*}(t)$ is, however, directly proportional to the spin concentration. An effective spin concentration can therefore be taken as $s c$ where $s$ is a scaling parameter. In essence, a "particle" in a Monte Carlo simulation can be considered to represent a collection of $s$ spins. By this means, both the spin density and $G^{*}(0)$ can be matched between MD and MC simultaneously.

The parametrization process, the values of the physical observables, and the MC simulation parameters are summarized in Table II. It was found that $s=4.0, b=0.239 \mathrm{~nm}$, and $\tau_{0}=3.13$ ps. These values were used to compute the correlation function from all MC simulations including those in 2D and Q2D.

\section{Boundary conditions}

The implementation of the boundary conditions for both MC and MD simulation requires some care as illustrated in Fig. 4. The figures illustrate a MC simulation of a Q2D system but the principles apply generally. The figure represents a slice through the system in the $x-z$ plane. The solid circles in Fig. 4(a) show the spins contained in the simulation cell at $t=0$. The system consists of the replication of the simulation cell in the $x$ and $y$ directions and the image spins are presented as open circles. The large circle (dashed line) indicates a sphere of radius $d$ (half the dimension of the simulation cell) centered on a specific spin. The spin at the center of the sphere interacts with all spins contained within this radius, that is, for $r_{0} \leqslant d$. It therefore interacts with an image spin contained in one of the replicated cells as illustrated by the vector $\mathbf{r}_{0,2}$ in the figure. This set of spin pairs is included in the calculation of the correlation function at all times, irrespective of how far the pair becomes separated.

Figure 4(b) illustrates the same system at a later time. The spins originally contained within the simulation cell at $t=0$ remain as solid circles. The correlation function is calculated using Eq. (11) for all pairs of spins for which $r_{0} \leqslant d$, that is, all spin pairs separated by a maximum distance $d$ at $t=0$. A typical spin pair is labeled $\mathbf{r}_{0,1}$ at $t=0$ and $\mathbf{r}_{1}$ at time $t$ and, in this case, both vectors have length less than $d$. This case is indicated in the figure by "NN" to indicate a "nearnear" pairing. For some spin pairs, of course, the diffusion process results in the spin pair vector $\mathbf{r}$ at time $t$ exceeding the distance $d$. This is illustrated by the vector labeled $\mathbf{r}_{2}$ where $r_{2}>d$. The correlation function is calculated using Eq. (11) for these pairs of spins too and this contributes to the "near-far" component of the correlation function. Also labeled in Fig. 4(b) is a third vector which constitutes a "far-near" contribution of the correlation function and is the time-reversed equivalent of the "near-far" vector in the illustration. All are included in the computation of the correlation function $G^{*}(t)$. The "far-far" contribution, when the interspin vector extends outside the sphere both initially and at time $t$, is not included.

\section{3. $3 D, 2 D$, and $Q 2 D$ simulations}

The 3D bulk-water MC simulation was undertaken using a $30 \times 30 \times 30$ simulation cell with periodic boundary conditions applied in all three directions. The diffusion coefficient was calculated from the gradient of $\left\langle r^{2}\right\rangle$ as a function of time and found to be $2.200 \pm 0.002 \times 10^{-9} \mathrm{~m}^{2} / \mathrm{s}$ as expected. The $2 \mathrm{D}$ MC simulation was undertaken using a $60 \times 60$ square lattice simulation cell.

The MC simulations of Q2D systems were undertaken using a cubic lattice with hard-wall boundaries operating in the $z$ direction as illustrated in Fig. 4. Simulations were executed for slab pores comprising 3, 5, 10, and 25 layers, approximately equivalent to thicknesses of $0.7,1.22 .4$, and $6.0 \mathrm{~nm}$, respectively.

\section{RESULTS AND DISCUSSION}

\section{A. MD results for bulk water}

The spin-pair dipolar correlation function $G_{\mathrm{MD}, 3 \mathrm{D}}^{*}(t)$ was determined from a MD simulation using Eq. (11) with statistical averaging over multiple time origins allowing the correlation function to be determined over the time interval 0-555 ps for the intramolecular and intermolecular spin pairs. The intramolecular spin relaxation is associated with the 


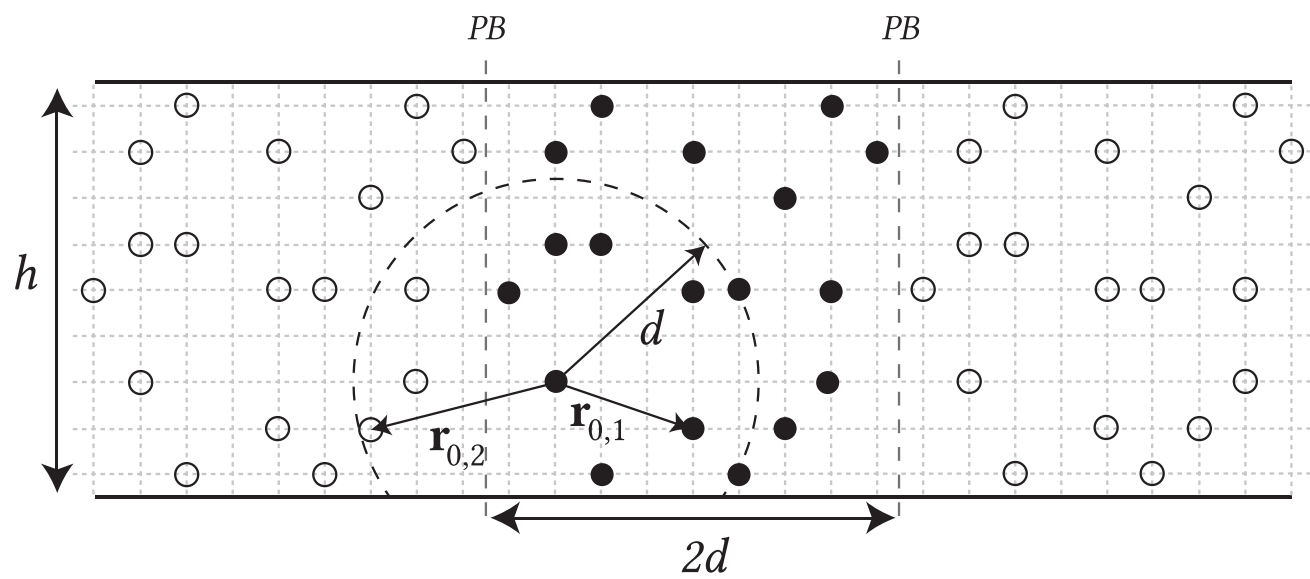

(a)

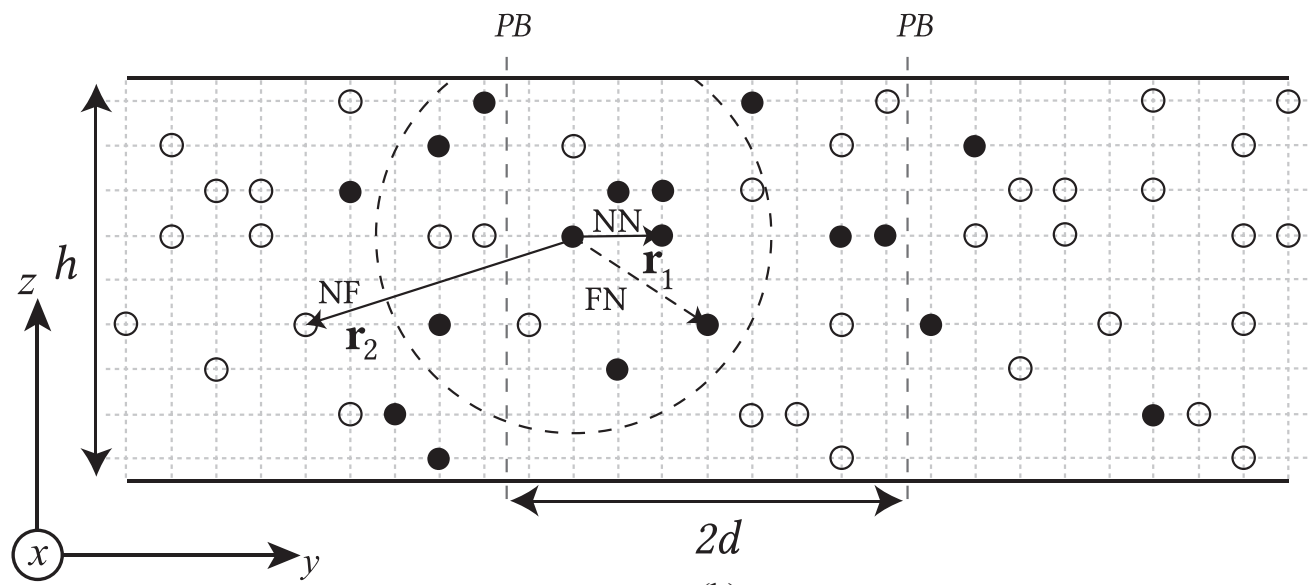

(b)

FIG. 4. A schematic diagram of a MC simulation of a Q2D spin system of thickness $h$ showing the spins in the simulation cell (a) at $t=0$ and (b) at a later time $t$ (filled circle). Periodic boundary conditions apply in the $x$ and $y$ directions (indicated by PB). The image spins are represented by open circles. In (a), all spin pairs contained within the sphere of radius $d$, such as the spin pairs labeled $\mathbf{r}_{0,1}$ and $\mathbf{r}_{0,2}$, are included in the computation of the correlation function. In (b), the spin pair labeled $\mathbf{r}_{1}$ is the pair labeled $\mathbf{r}_{0,1}$ in (a). This is labeled "NN" representing "near-near" because both vectors have a magnitude less than $d$. The spin pair labeled $\mathbf{r}_{2}$ is the pair labeled $\mathbf{r}_{0,2}$ in (a). This pair is labeled "NF" ("near-far") because $r_{2}>d$. Thus, the designation "F" or "N" depends on the vector length compared to $d$. Finally, the vector labeled "FN" is the time-reversed equivalent of $\mathbf{r}_{2}$.

tumbling motion of the water molecules in the magnetic field, whereas the intermolecular spin relaxation is associated with the relative translational diffusion of spins. The contributions to the intermolecular correlation function from spin pairs with separation $r_{0}<d$, where $d=2.475 \mathrm{~nm}$ (corresponding to half the simulation cell dimension), are obtained from the simulation. The correction to the simulated intermolecular correlation function $G_{\mathrm{FF}, 3 \mathrm{D}}^{*}(t)$, which accounts for spin pairs for which $r_{0}>d$ and $r>d$, is computed using Eq. (18) and added to the simulated intermolecular correlation function. The results are presented in Fig. 5, which shows that the correction term is less than $1 \%$ of the simulated intermolecular correlation function for $t<10 \mathrm{ps}$, about $10 \%$ at $t=45 \mathrm{ps}$ and exceeds the simulated intermolecular correlation function for $t>140 \mathrm{ps}$. At $t=140 \mathrm{ps}$, the root-mean-square distance moved by molecule is $1.85 \mathrm{~nm}$ so that the root-mean-square spin-pair separation is about $\sqrt{2} \times 1.85=2.6 \mathrm{~nm}$, approximately equal to $d$.

Equation (18) can be used to generate the correlation function $G_{\mathrm{u}, 3 \mathrm{D}}^{*}(t)$ for a uniform fluid by making an appropriate

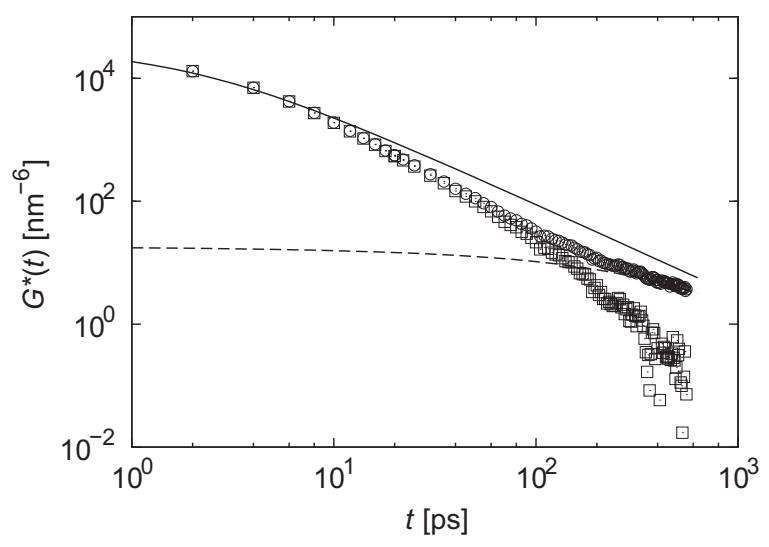

FIG. 5. The correlation function $G_{\mathrm{MD}, 3 \mathrm{D}}^{*}(t)$ is plotted as a function of time for MD simulation of bulk water (open square). The analytic correction term (dashed line) and the result for a uniform fluid are presented (solid line). The MD results with the correction term $G_{\mathrm{FF}, 3 \mathrm{D}}^{*}(\mathrm{t})$ added (open circle) are presented. 
choice for $\delta$ as described in Sec. IID. In this work, we set $\delta=0.19 \mathrm{~nm}$. This choice ensures that $G_{\mathrm{u}, 3 \mathrm{D}}^{*}(0)=G_{\mathrm{MD}, 3 \mathrm{D}}^{*}(0)$ as obtained from the MD simulation for the intermolecular correlation function. $G_{\mathrm{u}, 3 \mathrm{D}}^{*}(t)$ is also included in Fig. 5 where, as expected, very good agreement with the MD simulation results is found in the region $t<10 \mathrm{ps}$. There is also good agreement between $G_{\mathrm{FF}, 3 \mathrm{D}}^{*}(t)$ and $G_{\mathrm{u}, 3 \mathrm{D}}^{*}(t)$ for long times as the correlation function becomes increasingly dominated by the contribution due to spin pairs for which $r_{0}>d$ and $r>d$. We note also that the diffusion coefficient used in the computation of $G_{\mathrm{u}, 3 \mathrm{D}}^{*}(t)$ is the experimental value of $2.2 \times 10^{-9} \mathrm{~m}^{2} / \mathrm{s}$, whereas the value emerging from the MD simulation is $2.7 \times 10^{-9} \mathrm{~m}^{2} / \mathrm{s}$ as discussed in Sec. III A1.

Finally, we comment on the accuracy of the MD simulation approach for the purpose of determining the correlation function. The accuracy is limited by the physical dimensions of the simulation cell and the time scale of a simulation which, in turn, is restricted by computational resource. This is apparent in Fig. 5 where the contribution of the approximate correction term exceeds the simulated intermolecular correlation function for $t>140 \mathrm{ps}$. Grivet [23] undertook MD simulations for a Lennard-Jones fluid and discusses the limitations of MD in Sec. F of his paper in the context of water. Grivet points out that the dipolar relaxation scales as $r^{-2}$ and so a simulation cell size containing several hundred thousand spins is required if $99 \%$ of the contribution to the dipolar relaxation correlation function is to be obtained from the simulation.

In this work, the correlation function correction term $G_{\mathrm{FF}, 3 \mathrm{D}}^{*}(t)$ can be added to the MD simulation data resulting in substantially improved determination of the correlation function over the full time domain. This process reduces the need for a large simulation cell and a prohibitively large number of spins. The correction term is, however, an approximation which makes assumptions about the movement of spin pairs at longer times. It is therefore useful to establish a guide as to the relative impact of the correction term to the overall correlation function as a function of the MD simulation cell dimension. The correction term was therefore computed as a function of the distance $d$ (noting that the simulation cell size is equal to $2 d$ ) and compared to $G_{\mathrm{u}, 3 \mathrm{D}}^{*}(t)$. Figure 6 presents the time at which the correction term contributes more than

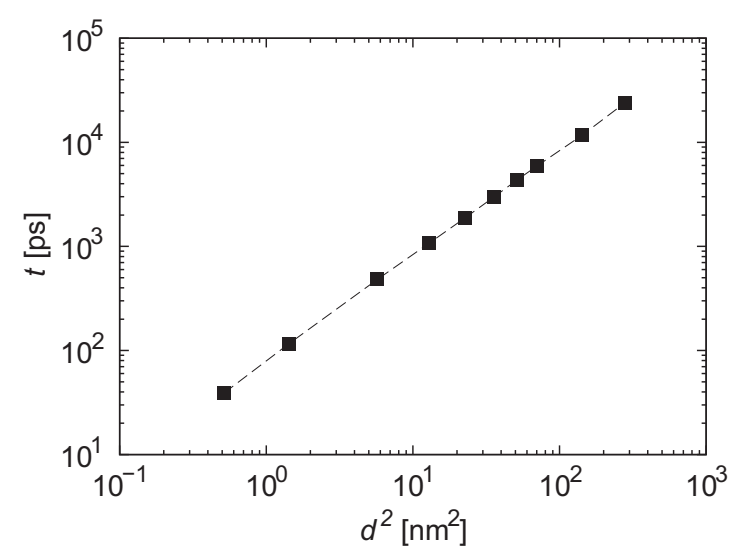

FIG. 6. The time for which $G_{\mathrm{FF}, 3 \mathrm{D}}^{*}(t)$ contributes more than $50 \%$ to the correlation function $G_{\mathrm{u}, 3 \mathrm{D}}^{*}(t)$ for a uniform fluid is plotted as a function of $d^{2}$ where $d$ is the spin-pair cutoff distance.

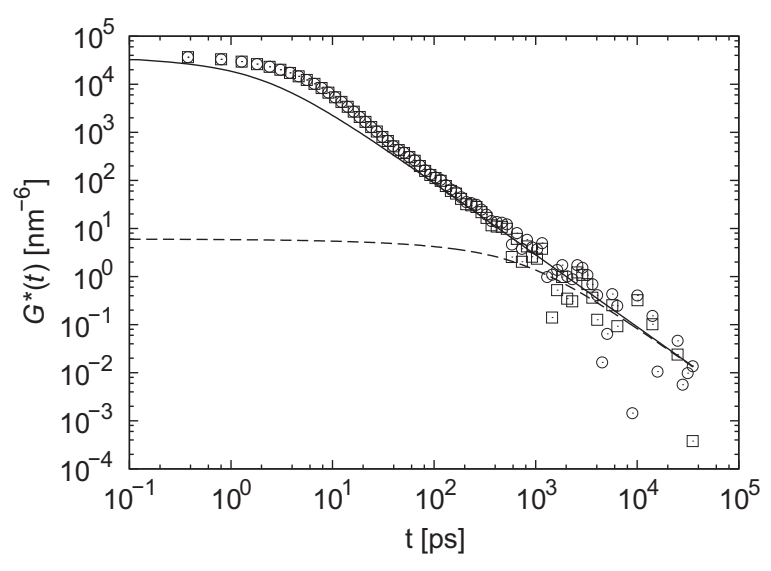

FIG. 7. The correlation function $G_{\mathrm{MC}, 3 \mathrm{D}}^{*}(t)$ from the MC simulation of bulk water is plotted as a function of time with (open circle) and without (open square) the correction term $G_{\mathrm{FF}, 3 \mathrm{D}}^{*}(t)$ added. The correction term $G_{\mathrm{FF}, 3 \mathrm{D}}^{*}(t)$ (dashed line) and the result for a uniform fluid are presented (solid line).

$50 \%$ to the correlation function for the uniform spin fluid. The time is presented as a function of $d^{2}$ yielding the expected linear relationship on a logarithmic scale. This figure provides a useful guide as to the impact of a chosen MD simulation cell dimension on the accuracy of the computation of the correlation function for practical cell dimensions.

\section{B. MC results for bulk water}

The MC simulation results for bulk water $G_{\mathrm{MC}, 3 \mathrm{D}}^{*}(t)$ are presented in Fig. 7 together with the correction to the correlation function $G_{\mathrm{FF}, 3 \mathrm{D}}^{*}(t)$, evaluated using Eq. (18) which has been added to the simulated data. The correction term was computed for $N_{v}=66.6$ spins $\mathrm{nm}^{-3}$ and the cutoff distance of $d=15 b=3.59 \mathrm{~nm}$. The correction term makes a small contribution to the total correlation function up to $t \gtrsim 1 \mathrm{~ns}$ because of the large cutoff distance. Beyond this time, the MC data are showing significant statistical scatter. The correlation function obtained from MC simulation is restricted to the translational motion of spins only.

The correlation function for the uniform fluid $G_{\mathrm{u}, 3 \mathrm{D}}^{*}(t)$ provides excellent agreement with the MC data at long times ( $t \gtrsim 100 \mathrm{ps}$ ). At shorter times $(t \lesssim 20 \mathrm{ps})$, there is a noticeable difference between the $\mathrm{MC}$ result and the uniform fluid. It is found that $G_{\mathrm{u}, 3 \mathrm{D}}^{*}(t)<G_{\mathrm{MC}, 3 \mathrm{D}}^{*}$ by a factor up to 2 . The correlation function is dominated at short times by pairs of spins that are nearest neighbors. The simulation results suggest that pairs of neighboring spins remain as neighbors for longer than predicted by the model of an uncorrelated uniform fluid. This may arise because, if one spin of a pair hops to a neighboring site, there is an enhanced probability (compared to $1-c$ ) of the other spin of the pair making a hop to the vacated site.

It is noted that $G_{3 \mathrm{D}}^{*}(t \rightarrow \infty)$ given by Eq. (25) (not shown in the figure) is in excellent agreement with $G_{\mathrm{u}, 3 \mathrm{D}}^{*}(t)$ for long times and is within $10 \%$ for times beyond $t \gtrsim 25$ ps. $G_{3 \mathrm{D}}^{*}(t \rightarrow$ $\infty)$ therefore provides a simple analytical expression for the correlation function for all but the shortest times.

Finally, Fig. 8 presents the correlation function for 3D bulk water obtained from the MD and MC simulations and the 


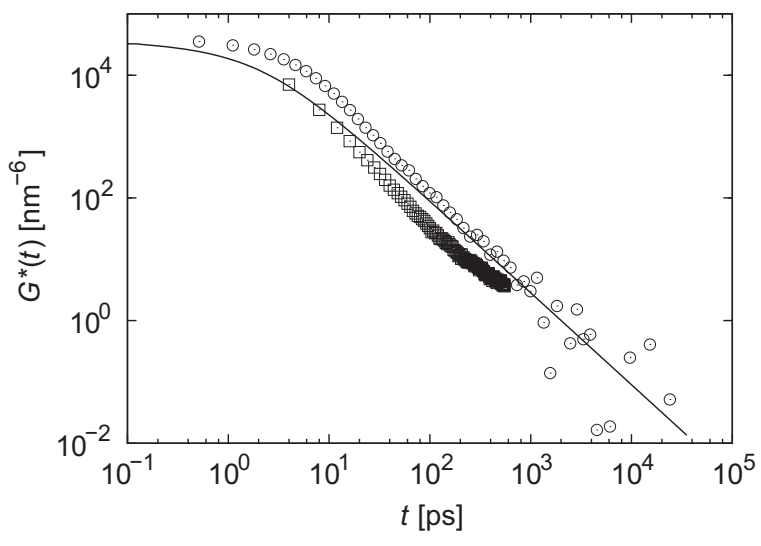

FIG. 8. The correlation function $G^{*}(t)$ is plotted as a function of time for the MD (open square) and MC (open circle) simulations of bulk water. The correction term $G_{\mathrm{FF}, 3 \mathrm{D}}^{*}$ has been added in both cases. The result for a uniform fluid is presented (solid line).

analytical theory for a uniform fluid. The agreement between $G_{\mathrm{u}, 3 \mathrm{D}}^{*}(t)$ and the MD results at short times and the MC results for long times is clearly apparent.

\section{MC results for a $2 \mathrm{D}$ system}

The results of MC simulations in 2D are now presented for spins confined to move on a $60 \times 60$ square lattice. The results for the correlation function $G_{\mathrm{MC}, 2 \mathrm{D}}^{*}(t)$ are presented in Fig. 9. The correction term was computed using Eq. (20) for $N_{s}=16.0$ spins $\mathrm{nm}^{-2}$ and a cutoff distance of $d=30 b=7.18 \mathrm{~nm}$. The correction term only makes a small contribution to the total correlation function because of the large cutoff distance.

The correlation function for the uniform fluid $G_{\mathrm{u}, 2 \mathrm{D}}^{*}(t)$ was computed according to Eq. (20) with $\delta=0.753 \mathrm{~nm}$. This choice ensures $G_{\mathrm{u}, 2 \mathrm{D}}^{*}(0)=G_{\mathrm{MC}, 2 \mathrm{D}}^{*}(0)$. Figure 9 shows that there is a good agreement with the MC data at long times

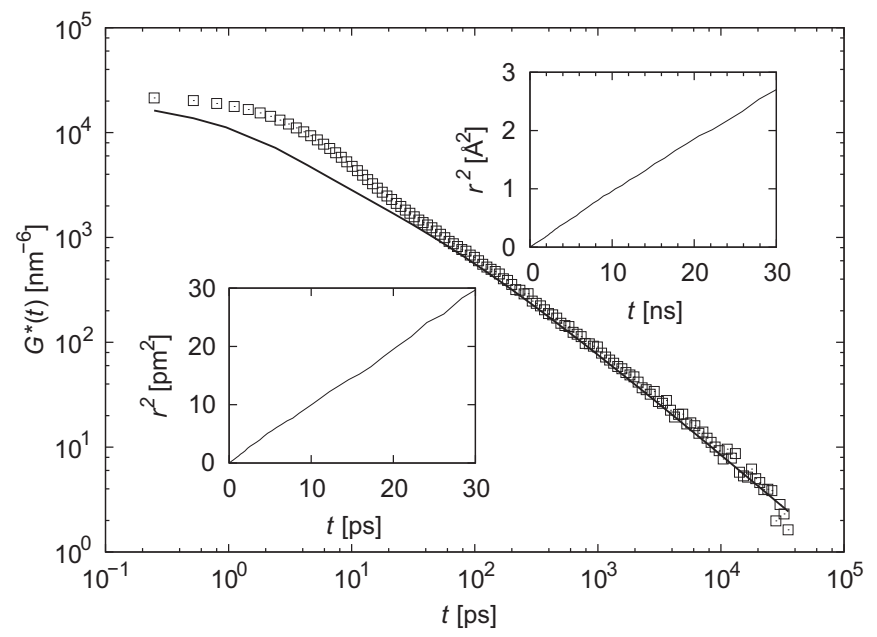

FIG. 9. The correlation function $G_{\mathrm{MC}, 2 \mathrm{D}}^{*}(t)$ is plotted as a function of time for MC simulation of water confined to a 2D $60 \times 60$ square lattice (open square). The result for a uniform $2 \mathrm{D}$ fluid is presented (solid line). The inserts show $\left\langle r^{2}\right\rangle$ as a function of time for the range 0-30 ps and 0-30 ns. where the difference is less than $10 \%$ at times longer than about 400 ps. It is found that $G_{\mathrm{u}, 2 \mathrm{D}}^{*}(t)<G_{\mathrm{MC}, 2 \mathrm{D}}^{*}(t)$ at short times $t \lesssim 20 \mathrm{ps}$, similar to the 3D results. Indeed, the difference is more pronounced in 2D. The difference is again associated with the correlated motion of spin pairs as described in the previous section where we note that the more pronounced deviation from the uniform fluid is consistent with the lower coordination number of the 2D square lattice.

The analytic expression valid for long times $G_{2 \mathrm{D}}^{*}(t \rightarrow \infty)$, given by Eq. (24) (not shown in the figure), provides an excellent representation of the MC data for $t \gtrsim 40 \mathrm{ps}$. We note, however, that $G_{2 \mathrm{D}}^{*}(t \rightarrow \infty)$ is larger than $G_{\mathrm{u}, 2 \mathrm{D}}^{*}(t)$ by about $15 \%-20 \%$. This is because $G_{2 \mathrm{D}}^{*}(t \rightarrow \infty)$ is determined for diffusion on a square lattice whereas $G_{\mathrm{u}, 2 \mathrm{D}}^{*}(t)$ is determined according to Eq. (20) for a model uniform fluid. Therefore, $G_{2 \mathrm{D}}^{*}(t \rightarrow \infty) \neq G_{\mathrm{u}, 2 \mathrm{D}}^{*}(t \rightarrow \infty)$ in $2 \mathrm{D}$, unlike in 3D where the two methods of estimating the long-time behavior of the correlation function agree.

Close examination of the MC data contained in Fig. 9 indicates a change in the gradient of the correlation function at about 15 ps. To establish if this was an effect associated with the finite size of the simulation cell, further simulations were undertaken for $30 \times 30$ and $15 \times 15$ square lattices. The change in gradient is apparent in all three systems at approximately the same time and therefore not associated with the finite size of the simulation cell. The abrupt change in gradient is not apparent in the uniform fluid. The inserts in the figure show that the diffusion coefficient (which is given by the gradient of $\left\langle r^{2}\right\rangle$ versus $t$ ) is very similar over short and longer time scales. There are no unusual correlation effects associated with the motion of individual particles at about 15 ps (which corresponds to an average of about 4 hops per spin). We speculate that the change in gradient in the correlation function is associated with the loss of the correlated motion of pairs of spins described earlier.

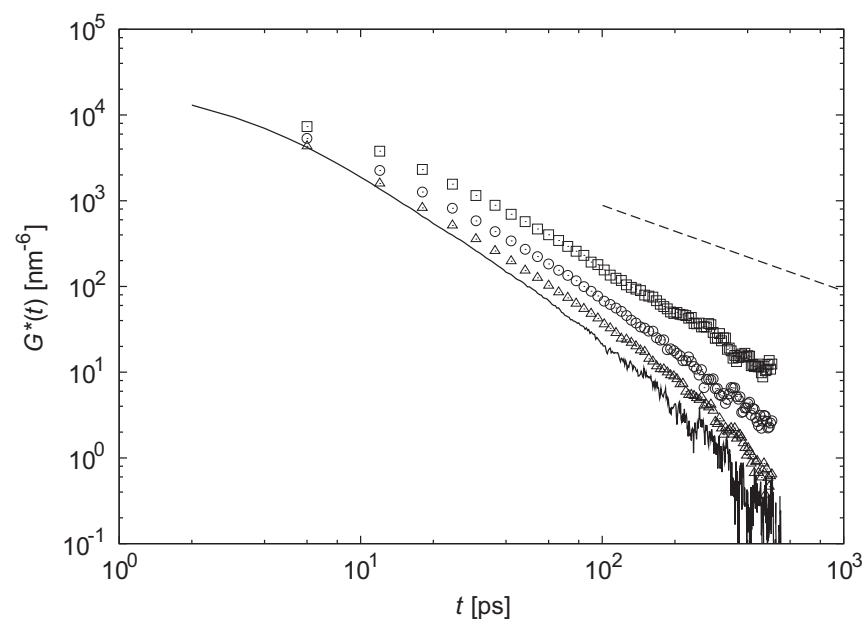

FIG. 10. The correlation function $G_{\mathrm{MD}, \mathrm{Q} 2 \mathrm{D}}^{*}(t)$ for the intermolecular spins is plotted as a function of time for MD simulations of water in a Q2D system with thickness approximately $1.0 \mathrm{~nm}$ (open square), $2.0 \mathrm{~nm}$ (open circle), and $5.0 \mathrm{~nm}$ (open triangle). The MD 3D data (solid line) and the analytic expression for $G_{\mathrm{Q} 2 \mathrm{D}}^{*}(t \rightarrow \infty)$ (dashed line) are also presented for comparison. 


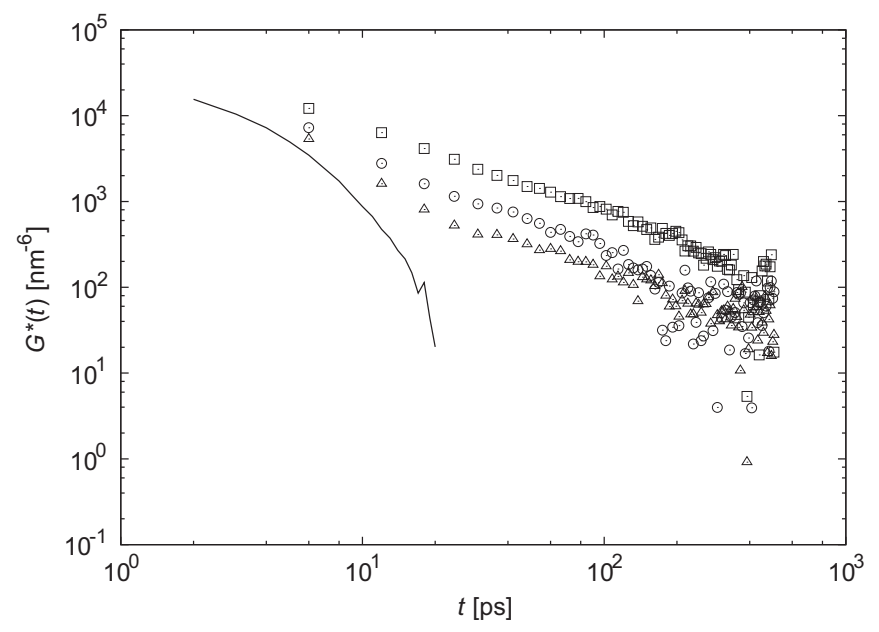

FIG. 11. The correlation function $G_{\mathrm{MD}, \mathrm{Q} 2 \mathrm{D}}^{*}(t)$ for the intramolecular spins is plotted as a function of time for MD simulations of water in a Q2D system with thickness approximately $1.0 \mathrm{~nm}$ (open square), $2.0 \mathrm{~nm}$ (open circle), and $5.0 \mathrm{~nm}$ (open triangle). The MD 3D data are also presented for comparison (solid line).

\section{MD results for Q2D systems}

The MD results for three Q2D systems are presented in Figs. 10 and 11 for the intermolecular and intramolecular spins, respectively. The simulation parameters are listed in Table I. Bulk-water results are also included for reference. No correction term has been added to the MD simulation data for the Q2D systems and, for the purpose of comparison, $G_{\mathrm{FF}, \mathrm{MD}}^{*}$ has also not been added to the MD results for bulk water.
The water density function determined as a function of $z$ (not presented) indicates that the water density is nonuniform over a distance of about $0.5 \mathrm{~nm}$. This structuring arises due to the specific interactions of water molecules with the surface and steric effects associated with surface roughness. The diffusion and rotation of the water close to the surfaces are restricted. Consequently, the volume of water which can be considered to be "bulk water" in the Q2D systems is less than suggested by the thickness of the pore. Figure 10 shows that the correlation function for water is tending to that of bulk water as the pore thickness increases. No distinctive transition from $3 \mathrm{D}$ to $2 \mathrm{D}$ diffusive behavior is observed in the MD correlation function on the time scale of the simulations. $G_{\mathrm{Q} 2 \mathrm{D}}^{*}(t \rightarrow \infty)$ is also presented, as given by Eq. (30), and shows that the simulation time scale is too short to observe $t^{-1}$ dependence of the correlation function. Indeed, we speculate that the diffusion process may be characteristic of a Lévy process in Q2D systems with adsorption at the surfaces interspersed with occasional bulk-water excursions [11].

Figure 11 shows the intramolecular component of the spin correlation function. This contribution is associated with relaxation due to the tumbling motion of the molecular spins in the static magnetic field. There is a large departure from the 3D bulk intramolecular correlation function for all Q2D systems. This is due to the orientation of water molecules bound to surface sites and structured water affected by the surface. The potential energy for the water at the surface leads to preferred orientations of the molecules and therefore the correlation function reflects the persistence of these correlations over large times.

The value of $T_{1}$ for water was calculated using Eqs. (1a), (2), and (5) for both the intermolecular (translational) and intramolecular (rotational) spin components, and the results (a)

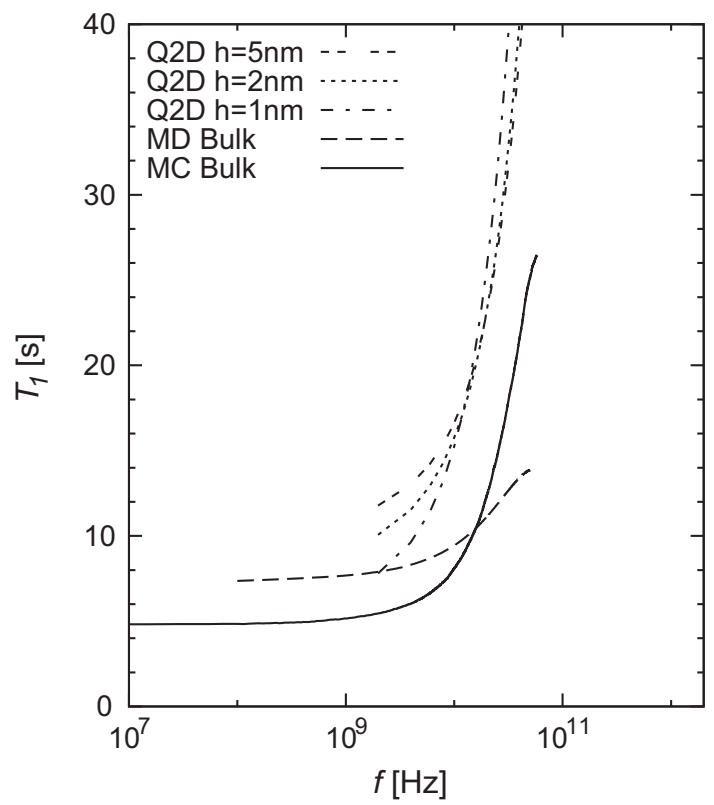

(b)

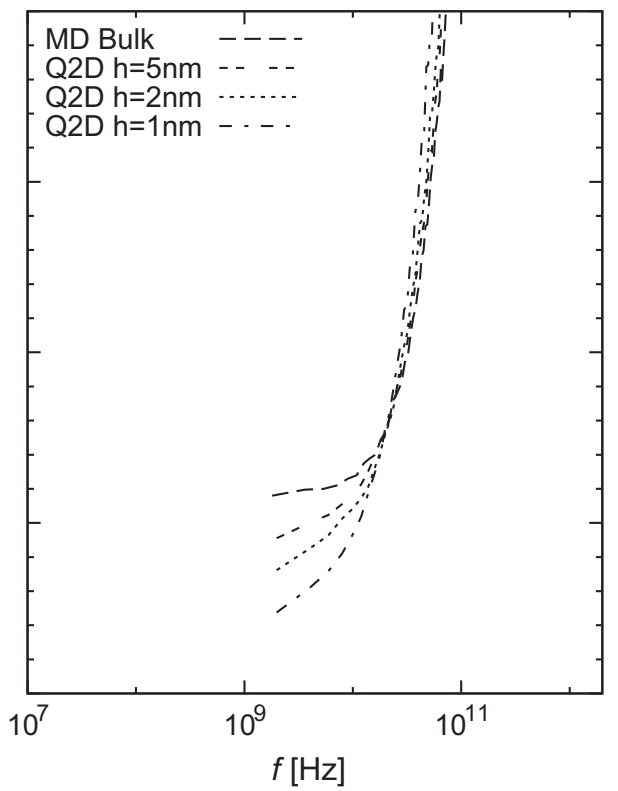

FIG. 12. The component of the spin-lattice relaxation time $T_{1}$ is calculated for (a) the intermolecular spin interactions and (b) the intramolecular spin interactions as a function of frequency. These are associated with the translational and rotational motion of spins, respectively. The overall rate is given by $T_{1}^{-1}=T_{1, \text { trans }}^{-1}+T_{1, \text { rot }}^{-1}$. 
are presented in Fig. 12. The results for $h=1.5 \mathrm{~nm}$ are omitted for clarity. $T_{1}$ associated with the intermolecular spins is found to be a weak function of frequency at low frequencies with a value $7.4 \mathrm{~s}$ at about $150 \mathrm{MHz}$. Note that the correction term $G_{\mathrm{FF}, 3 \mathrm{D}}^{*}$ has been added to the MD simulation data for bulk water, enabling $T_{1}$ to be determined at the lower frequencies.

The value of $T_{1}$ obtained for the intramolecular spins is difficult to determine in the lower frequency range due to the limited time scale of the MD simulations and the absence of a longtime correction term for the rotational component of the correlation function. Extrapolation to lower frequencies of the data extending to $1 \mathrm{GHz}$ for bulk water suggests that $T_{1} \approx 10.0 \pm$ $1.0 \mathrm{~s}$ at about $150 \mathrm{MHz}$. The overall rate is given by $T_{1}^{-1}=$ $T_{1, \text { trans }}^{-1}+T_{1, \text { rot }}^{-1}$ yielding $4.3 \pm 0.4 \mathrm{~s}$ in broad agreement with the experimental value of $3.8 \mathrm{~s}$ at $300 \mathrm{~K}$ for bulk water [42]. Figure 12(b) indicates that $T_{1}$ at low frequencies for all Q2D systems is significantly shorter than the result for bulk water.

\section{E. MC results for Q2D systems}

The MC simulations were undertaken for Q2D pores comprising $n=3,5,10$, and 25 layers as listed in Table I. The correlation function arising due to translational motion of the spins is presented in Fig. 13 (the results for 10 layers are omitted for clarity). The $3 \mathrm{D}$ data are also plotted for comparison. No correction term is added to the correlation function presented in Fig. 13. Also included in Fig. 13 is $G_{\mathrm{Q} 2 \mathrm{D}}^{*}(t \rightarrow \infty)$ as given by Eq. (30) (the line is similar on a logarithmic plot for all values of $n$ ). The MC results for $n=3$ are clearly converging to $G_{\mathrm{Q} 2 \mathrm{D}}^{*}(t \rightarrow \infty)$. The results for 25 layers are similar to results for $3 \mathrm{D}$ bulk water over the full length of the simulation as shown in Fig. 13.

The results in Fig. 13 demonstrate a clear transition from 3D diffusive behavior at short times to $2 \mathrm{D}$ behavior at longer times with the transition time dependent on the layer thickness. The time at which the correlation function moves from 3D to 2D behavior $t_{3 \mathrm{D} \rightarrow 2 \mathrm{D}}$ has been estimated and is plotted as a function

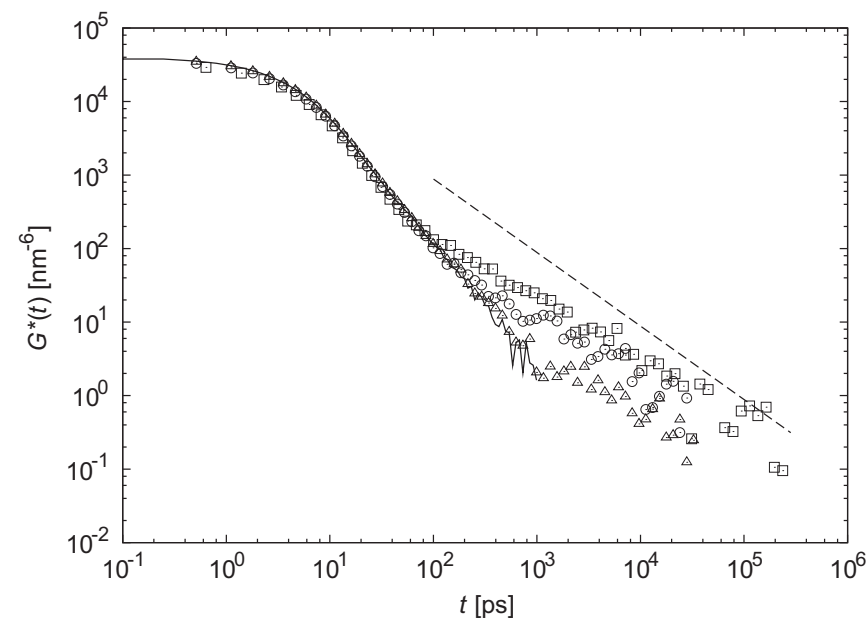

FIG. 13. The correlation function $G^{*}(t)$ is plotted as a function of time for MC simulations of water in a Q2D system with thickness 3 layers $(0.7 \mathrm{~nm})$ (open square), 5 layers $(1.2 \mathrm{~nm})$ (open circle), and 25 layers $(6.0 \mathrm{~nm})$ (open triangle). The MC 3D data (solid line) and the analytic expression for $G_{\mathrm{Q} 2 \mathrm{D}}^{*}(t \rightarrow \infty)$ (dashed line) are also presented for comparison.

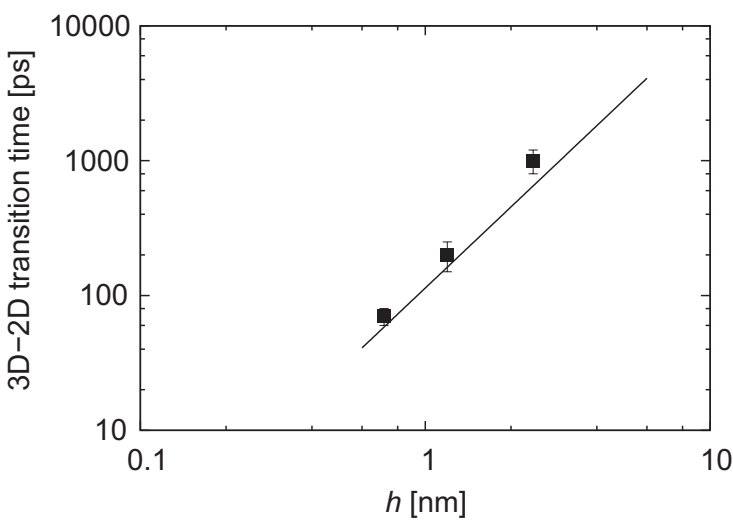

FIG. 14. The approximate time at which water moves from 3D to 2D behavior is plotted as a function of the Q2D pore thickness $h$. The solid line is the average time for a pair of unrestricted spins to move apart by a distance $h$ in the $z$ direction.

of layer thickness $h$ in Fig. 14. The gradient is approximately equal to 2 , consistent with the expectation that $t_{3 \mathrm{D} \rightarrow 2 \mathrm{D}}$ depends on the square of a distance. Also plotted in Fig. 14 is the curve assuming that $t_{3 \mathrm{D} \rightarrow 2 \mathrm{D}}$ is the time taken for a pair of unrestricted spins to separate by a distance $h$ in the $z$ direction. The physical basis is that a spin pair will have been influenced by the boundaries and change to $2 \mathrm{D}$ diffusive behavior once the change in separation in the $z$ direction exceeds the thickness $h$. This curve shows good agreement with the simulation data.

Finally, $T_{1}$ was calculated at low frequencies and found to be very similar to the $3 \mathrm{D}$ bulk-water result of $4.8 \mathrm{~s}$ at $15 \mathrm{MHz}$ for each layer thickness. Although the MC results behave as expected, the Q2D systems do not include the complexity of the MD simulations due to the noninteracting hard walls.

\section{CONCLUSION}

MD and MC simulations of water dynamics, operating at different length and time scales, have been used to determine the ${ }^{1} \mathrm{H}$ dipolar spin-pair correlation function, required for the calculation of nuclear spin relaxation times, in 3D, 2D, and Q2D systems. The systems chosen for analysis are simple and designed to establish the numerical simulation methodology. They also establish the constraints of numerical modeling techniques for the study of water in confined geometries. The extent to which analytical models based on a uniform spin fluid can contribute to the understanding and accuracy of the results is established. More complex simulations exploring the effect of immobile spins on surfaces, the orientational ordering of water at surfaces, paramagnetic impurities, geometrical effects, and so forth can then be undertaken with the full knowledge of the constraints that operate. We demonstrate that MD simulation is a powerful method of elucidating the diffusive behavior of spins on the atomistic scale. Results can be used to devise more accurate models for the motion of spins. In this work, the MD simulations of bulk water are used to parametrize MC simulations which can access longer time scales and larger simulation cell sizes.

In this paper, we have demonstrated that the expression (11) presented by Sholl [24] for determining the correlation 
function for powder samples, originally developed for 3D systems, is also applicable for 2D and Q2D spin systems. This constitutes an efficient means of extracting powder-average correlation functions from numerical simulations. We present expressions for the correction term $G_{\mathrm{FF}}^{*}(t)$ for $3 \mathrm{D}$ and $2 \mathrm{D}$ systems. The correction term is added to the correlation function obtained from the MD and MC simulations to account for the contribution of spin pairs with separation greater than a cutoff distance $d$ at both $t=0$ and at time $t$. This term is derived assuming a uniform fluid and is shown to improve the accuracy of the simulation results for long times. The equivalent correction term is not available for Q2D systems, but we derive simple analytic expressions for $G^{*}(t \rightarrow \infty)$ for spins diffusing on 3D, 2D, and Q2D lattices.

Analytic expressions for $G^{*}(t)$ for a uniform spin fluid are derived in $3 \mathrm{D}$ and $2 \mathrm{D}$ and serve as a useful reference system for comparison to the simulations. The expressions are the same as for the correction terms but with a cutoff distance $\delta$ which can be chosen to yield the value of $G^{*}(0)$ obtained from the simulation. The results from the MD and MC simulations for the correlation function for bulk water demonstrate that the uniform fluid constitutes a good model for $t \gtrsim 40$ ps. Quantities derived from the 3D simulations, such as the diffusion coefficient and the spin-lattice relaxation time $T_{1}$, are in very good agreement with experimental results for bulk water.

$G^{*}(t)$ obtained from MC simulations of Q2D systems, where water is confined by hard walls, is found to execute a distinct transition from 3D to 2D behavior. The transition time is found to be approximately the time taken for a pair of unrestricted spins to move apart in the $z$ direction by the thickness $h$ of the pore. The $T_{1}$ obtained from MC simulations of Q2D systems are found to be similar, independent of $h$, and equal to about $4.8 \mathrm{~s}$.

The $T_{1}$ obtained from MD simulations of Q2D systems of thickness $1-5 \mathrm{~nm}$, in which water is confined by $\left(\begin{array}{lll}1 & 0 & 0\end{array}\right)$ alpha-quartz surfaces, are presented for the intermolecular and intramolecular components. These correspond to the relaxation associated with the translational and rotational motion of the spins, respectively. The correlation function and hence $T_{1}$ are significantly changed due to the orientation and adsorption of spins at the surfaces. While the results for $T_{1}$ only allow access to very high frequencies due to the limited time scale of the simulations, the results clearly indicate that the relaxation time is substantially shorter in the Q2D systems.

\section{ACKNOWLEDGMENTS}

N.C.H. and J.S.B. acknowledge the support of Engineering and Physical Sciences Research Council (UK) for financial support (Grant No. EP/H033343/1).

\section{APPENDIX A: EXPANSION COEFFICIENTS}

This Appendix describes the calculation of the expansion coefficients $d_{m M}$ appearing in the expressions (7) and (8). These enable the spherical harmonic functions in one frame of reference to be directly calculated from the known spherical harmonic functions in a second frame. In general,

$$
Y_{2 m}(\theta, \phi)=\sum_{M=-2}^{2} d_{m M} Y_{2 m}\left(\theta^{\prime}, \phi^{\prime}\right),
$$

where the spherical harmonic functions $Y_{2 m}(\theta, \phi)$ in the laboratory frame $C$ are obtained for each spin-pair vector which has spherical harmonic functions $Y_{2 m}\left(\theta^{\prime}, \phi^{\prime}\right)$ evaluated in the $C^{\prime}$ frame.

The method used is taken from Romanowski and Krukowski [29]. The $d_{m M}$ are the matrix elements of a $5 \times 5$ matrix $\mathbf{D}_{2}$, where the subscript indicates that the consideration is restricted to the second order spherical harmonic functions. $\mathbf{D}_{\mathbf{2}}$ is expressed as the product of three matrices:

$$
\mathbf{D}_{2}=\left(\begin{array}{cccc}
d_{-2-2} & d_{-2-1} & d_{-20} & \cdots \\
d_{-1-2} & d_{-1-1} & \cdots & \\
\cdots & & &
\end{array}\right)=\mathbf{A}_{\mathbf{2}}^{-\mathbf{1}} \mathbf{C}_{\mathbf{2}} \mathbf{A}_{\mathbf{2}}
$$

where

$$
\begin{gathered}
\mathbf{A}_{2}=\sqrt{\frac{2 \pi}{15}}\left(\begin{array}{ccccc}
0 & i & 0 & i & 0 \\
0 & 1 & 0 & -1 & 0 \\
i & 0 & 0 & 0 & -i \\
2 & 0 & 0 & 0 & 2 \\
0 & 0 & 2 \sqrt{6} & 0 & 0
\end{array}\right), \\
\mathbf{A}_{2}^{-1}=\sqrt{\frac{15}{2 \pi}}\left(\begin{array}{ccccc}
0 & 0 & \frac{-i}{2} & \frac{1}{4} & 0 \\
\frac{-i}{2} & \frac{1}{2} & 0 & 0 & 0 \\
0 & 0 & 0 & 0 & \frac{\sqrt{6}}{12} \\
\frac{-i}{2} & -\frac{1}{2} & 0 & 0 & 0 \\
0 & 0 & \frac{i}{2} & \frac{1}{4} & 0
\end{array}\right) .
\end{gathered}
$$

The coordinate frame $C^{\prime}$ is obtained by rotating the frame $C$ anticlockwise about a vector $\mathbf{u}$ (as viewed looking along $\mathbf{u}$ from its positive end towards the origin) through an angle $\alpha$. The unit vector $\mathbf{u}=\left(u_{x}, u_{y}, u_{z}\right)$ has components measured in the frame $C$. The matrix $\mathbf{C}_{\mathbf{2}}$ in Eq. (A2) contains the information about the rotation.

Suppose $\mathbf{r}$ is a vector with coordinates expressed in frame $C$ as $\mathbf{r} \equiv(x, y, z) \equiv(r, \theta, \phi)$ and in frame $C^{\prime}$ as $\left(x^{\prime}, y^{\prime}, z^{\prime}\right) \equiv$ $\left(r, \theta^{\prime}, \phi^{\prime}\right)$. The coordinates of $\mathbf{r}$ in $C$ can be obtained by

$$
\left(\begin{array}{l}
x \\
y \\
z
\end{array}\right)=\mathbf{R}(\mathbf{u},-\alpha)\left(\begin{array}{c}
x^{\prime} \\
y^{\prime} \\
z^{\prime}
\end{array}\right),
$$

where the rotation matrix

$$
\mathbf{R}(\mathbf{u},-\alpha)=\left(\begin{array}{ccc}
r_{1} & r_{2} & r_{3} \\
r_{4} & r_{5} & r_{6} \\
r_{7} & r_{8} & r_{9}
\end{array}\right)=\left(\begin{array}{ccc}
u_{x}^{2}+\left(1-u_{x}^{2}\right) c & u_{x} u_{y}(1-c)-u_{z} s & u_{z} u_{x}(1-c)+u_{y} s \\
u_{x} u_{y}(1-c)+u_{z} s & u_{y}^{2}+\left(1-u_{y}^{2}\right) c & u_{y} u_{z}(1-c)-u_{x} s \\
u_{z} u_{x}(1-c)-u_{y} s & u_{y} u_{z}(1-c)+u_{x} s & u_{z}^{2}+\left(1-u_{z}^{2}\right) c
\end{array}\right)
$$


where $c=\cos \alpha$ and $s=\sin \alpha$. The matrix $\mathbf{C}_{2}$ is then given by Romanowski and Krukowski [29] in Eq. (35) of their paper. Unfortunately, this matrix has columns 4 and 5 switched. The correct expression for $\mathbf{C}_{2}$ is

$$
\mathbf{C}_{2}=\left(\begin{array}{ccccc}
r_{6} r_{8}+r_{5} r_{9} & r_{6} r_{7}+r_{4} r_{9} & r_{5} r_{7}+r_{4} r_{8} & r_{4} r_{7}+\frac{1}{2} r_{6} r_{9} & \frac{1}{2} r_{6} r_{9} \\
r_{3} r_{8}+r_{2} r_{9} & r_{3} r_{7}+r_{1} r_{9} & r_{2} r_{7}+r_{1} r_{8} & r_{1} r_{7}+\frac{1}{2} r_{3} r_{9} & \frac{1}{2} r_{3} r_{9} \\
r_{3} r_{5}+r_{2} r_{6} & r_{3} r_{4}+r_{1} r_{6} & r_{2} r_{4}+r_{1} r_{5} & r_{1} r_{4}+\frac{1}{2} r_{3} r_{6} & \frac{1}{2} r_{3} r_{6} \\
v_{1} & v_{2} & v_{3} & v_{4} & v_{5} \\
w_{1} & w_{2} & w_{3} & w_{4} & w_{5}
\end{array}\right),
$$

where

$$
\begin{aligned}
v_{1} & =2\left(r_{2} r_{3}-r_{5} r_{6}\right), \quad w_{1}=4 r_{8} r_{9}-2\left(r_{2} r_{3}+r_{5} r_{6}\right), \\
v_{2} & =2\left(r_{1} r_{3}-r_{4} r_{6}\right), \quad w_{2}=4 r_{7} r_{9}-2\left(r_{1} r_{3}+r_{4} r_{6}\right), \\
v_{3} & =2\left(r_{1} r_{2}-r_{4} r_{5}\right), \quad w_{3}=4 r_{7} r_{8}-2\left(r_{1} r_{2}+r_{4} r_{5}\right), \\
v_{4} & =r_{1}^{2}-r_{4}^{2}+\frac{1}{2}\left(r_{3}^{2}-r_{6}^{2}\right), \\
w_{4} & =r_{9}^{2}-r_{1}^{2}-r_{4}^{2}+2 r_{7}^{2}-\frac{1}{2}\left(r_{3}^{2}+r_{6}^{2}\right), \\
v_{5} & =\frac{1}{2}\left(r_{3}^{2}-r_{6}^{2}\right), \quad w_{5}=r_{9}^{2}-\frac{1}{2}\left(r_{3}^{2}+r_{6}^{2}\right) .
\end{aligned}
$$

The calculation proceeds as follows. The rotation matrix $\mathbf{R}(\mathbf{u},-\alpha)$ is determined (this may involve more than one rotation via the product of individual rotation matrices) and its components are used to determine the $5 \times$ 5 matrix $\mathbf{C}_{2}$ using Eq. (A6). Executing the matrix multiplications given by Eq. (A2) yields the the expansion coefficients $d_{m M}$.

\section{APPENDIX B: DETERMINATION OF $G_{\mathrm{FF}, 2 \mathrm{D}}^{*}$}

The starting point for the derivation of the correction term $G_{\mathrm{FF}, 2 \mathrm{D}}^{*}$ is Eq. (13) in which the summation of spin pairs is replaced by an integral over appropriate limits as follows:

$$
\begin{aligned}
G_{\mathrm{FF}, 2 \mathrm{D}}^{*}(t)= & \frac{4 \pi N_{s}}{5} \int_{d}^{\infty} \int_{d}^{\infty}\left[\sum_{M=-2}^{2} \frac{Y_{2 M}\left(\frac{\pi}{2}, \phi_{0}^{\prime}\right) Y_{2 M}^{*}\left(\frac{\pi}{2}, \phi^{\prime}\right)}{r_{0}^{3} r^{3}}\right] \\
& \times P\left(\mathbf{r}, t \mid \mathbf{r}_{0}\right) d^{\prime 2} \mathbf{r}_{0} d^{\prime 2} \mathbf{r},
\end{aligned}
$$

where $P\left(\mathbf{r}, t \mid \mathbf{r}_{0}\right)$ is the probability that a pair of spins is separated by $\mathbf{r}$ at time $t$ given the spin pair was separated by $\mathbf{r}_{0}$ at $t=0$ and is given by Eq. (17) with $n=2$. Note that this expression uses the conditional probability $P\left(\mathbf{r}, t \mid \mathbf{r}_{0}\right)$ and assumes that the a priori probability that a spin is at $\mathbf{r}_{0}$ at $t=0$ is given by the radial probability density function which is assumed to be unity at distances greater than the cutoff $d$ consistent with the assumption of an isotropic fluid. The prime indicates that the angular coordinates of the vectors are measured in the simulation frame $C^{\prime}$. The lower limit on the integrals $d$ can reasonably be chosen as half the length of the simulation cell.
Equation (17) is substituted into Eq. (B1) and then replaced by its well-known Fourier integral to yield

$$
\begin{aligned}
G_{\mathrm{FF}, 2 \mathrm{D}}^{*}(t)= & \frac{4 \pi N_{s}}{20 \pi^{2}} \int_{d}^{\infty} \int_{d}^{\infty}\left[\sum_{M=-2}^{2} \frac{Y_{2 M}\left(\frac{\pi}{2}, \phi_{0}^{\prime}\right) Y_{2 M}^{*}\left(\frac{\pi}{2}, \phi^{\prime}\right)}{r_{0}^{3} r^{3}}\right] \\
& \times\left[\int_{0}^{\infty} e^{-2 D t k^{2}} e^{i \mathbf{k} \cdot \mathbf{r}} e^{-i \mathbf{k} \cdot \mathbf{r}_{0}} d^{\prime 2} \mathbf{k}\right] d^{\prime 2} \mathbf{r}_{0} d^{\prime 2} \mathbf{r} .
\end{aligned}
$$

The exponential functions can be replaced using the JacobiAnger expression

$$
e^{i \mathbf{k} \cdot \mathbf{r}}=\sum_{n=-\infty}^{\infty} i^{n} J_{n}(k r) e^{i n \phi^{\prime}} e^{-i n \phi_{k}^{\prime}}
$$

where the azimuthal angles for $\mathbf{r}$ and $\mathbf{k}$ in the simulation frame are $\phi^{\prime}$ and $\phi_{k}^{\prime}$, respectively. The $J_{n}$ are Bessel functions of integer order. Equation (B3), and the well-known expressions for the spherical harmonic functions (which are nonzero for $M=0, \pm 2$ ), are substituted into Eq. (B2). Using $d^{\prime 2} \mathbf{r}=$ $r d r d \phi^{\prime}$, the integration over azimuthal angle $\phi^{\prime}$ may be undertaken explicitly noting that the integral is zero except for the combinations $(n=2, M=-2),(n=-2, M=2)$, and $(n=0, M=0)$.

The identical procedure is followed for the integration over angle $\phi_{0}^{\prime}$ whereupon the expression reduces to

$$
\begin{aligned}
G_{\mathrm{FF}, 2 \mathrm{D}}^{*}(t)= & \frac{4 \pi N_{s}}{16 \pi} \int_{0}^{\infty} e^{-2 D t k^{2}} \int_{d}^{\infty} \int_{d}^{\infty} \\
& \times\left[\frac{3 J_{2}(k r) J_{2}\left(k r_{0}\right)+J_{0}(k r) J_{0}\left(k r_{0}\right)}{r_{0}^{2} r^{2}}\right] d r_{0} d r d^{\prime 2} \mathbf{k},
\end{aligned}
$$

where the identity $J_{-n}(x)=J_{n}(x)$ has been used. Recognizing that $d^{\prime 2} \mathbf{k}=k d k d \phi_{k}^{\prime}$, the integration over $\phi_{k}^{\prime}$ is trivial and equal to $2 \pi$. It is also evident that the integrations with respect to $r_{0}$ and $r$ are identical. Equation (B4) now reduces to

$$
\begin{aligned}
G_{\mathrm{FF}, 2 \mathrm{D}}^{*}(t)= & \frac{4 \pi N_{s}}{8} \int_{0}^{\infty} k e^{-2 D t k^{2}}\left(3\left[\int_{d}^{\infty} \frac{J_{2}(k r)}{r^{2}} d r\right]^{2}\right. \\
& \left.+\left[\int_{d}^{\infty} \frac{J_{0}(k r)}{r^{2}} d r\right]^{2}\right) d k,
\end{aligned}
$$

which is the final expression for the $2 \mathrm{D}$ correction term accounting for spin pairs for which $r_{0}>d$ and $r>d$. 


\section{APPENDIX C: DETERMINATION OF THE LATTICE SUMMATION $L_{Q 2 D}$}

The lattice summation for Q2D systems is required for Eq. (30) which provides a simple analytic form for the correlation function for times sufficiently long that the diffusion may be described as 2D and the spin probability density in the $z^{\prime}$ direction (the direction of confinement) is uniform. The lattice summation $L_{\mathrm{Q} 2 \mathrm{D}}$ is given by the expression

$$
L_{\mathrm{Q} 2 \mathrm{D}}=\frac{1}{n} \sum_{i} \frac{1}{\rho_{i}^{3}} \sum_{j} \frac{P_{2}\left(\cos \psi_{i j}\right)}{\rho_{j}^{3}} .
$$

The lattice summation is executed for a Q2D system comprising $n$ layers where each layer consists of an infinite sheet of lattice sites arranged in a square lattice.

Care is required in the computation of Eq. (C1) for a Q2D lattice. Consider the model of a slab pore containing $n=3$ layers so that each of the 3 layers comprises an infinite square lattice, labeled $\ell=0,1,2$. Each layer clearly connects with a different set of lattice vectors. For example, a lattice point on layer $\ell=0$ has a lattice vector located at $(0,0,1)$ unlike a lattice point in layer $\ell=2$ due to the presence of the hard-wall boundary. The set of lattice vectors associated with a lattice point in layer $\ell$ consists of the sum of $n$ layers which can be labeled according to the $z$ displacement of the layer via an index $k$. Therefore, layer $\ell=0$ has possible $k$ values of 0,1 , and 2 , layer $\ell=1$ has $k$ values of $-1,0$, and 1 , and layer $\ell=2$ has $k$ values of $-2,-1$, and 0 . The lattice summation becomes the average over the $n$ layers. The total set of lattice vectors comprises a summation over both $\ell$ and $k$. Similarly, therefore, the double summation represented by Eq. (C1) must incorporate all possible combinations of $(\ell, k)$ and $\left(\ell^{\prime}, k^{\prime}\right)$.

Equation (C1) therefore becomes

$$
\begin{aligned}
L_{\mathrm{Q} 2 \mathrm{D}} & =\frac{1}{n^{2}} \sum_{\ell=0}^{n-1} \sum_{k=-\ell}^{\ell+n-1} \sum_{i} \frac{1}{\rho_{k i}^{3}} \sum_{\ell^{\prime}=0}^{n-1} \sum_{k^{\prime}=-\ell^{\prime}}^{\ell^{\prime}+n-1} \sum_{j} \frac{P_{2}\left(\cos \psi_{i j}\right)}{\rho_{k^{\prime} j}^{3}} \\
& =\frac{1}{n^{2}} \sum_{\ell=0}^{n-1} \sum_{k=-\ell}^{\ell+n-1} \sum_{\ell^{\prime}=0}^{n-1} \sum_{k^{\prime}=-\ell^{\prime}}^{\ell^{\prime}+n-1} L_{\mathrm{Q} 2 \mathrm{D}}\left(k, k^{\prime}\right),
\end{aligned}
$$

TABLE III. The value of the lattice constant $L_{\mathrm{Q} 2 \mathrm{D}}$ is presented for Q2D systems comprising $n$ layers of square lattice.

\begin{tabular}{cc}
\hline \hline$n$ & $L_{\mathrm{Q} 2 \mathrm{D}}$ \\
\hline 1 & 20.402 \\
2 & 18.922 \\
3 & 18.427 \\
4 & 18.180 \\
5 & 18.032 \\
10 & 17.735 \\
$\infty$ & 17.438 \\
\hline \hline
\end{tabular}

where the summations over $i$ and $j$ are over the set of planar lattice vectors and where the notation $\rho_{k i}$ refers to the planar lattice vectors offset in the $z$ direction by $k$.

On this basis, taking a three-layer slab pore $(n=3)$ as the working example, 81 lattice summations are required. However, symmetry relationships significantly reduce the number of separate lattice summations required. For example, $L_{\mathrm{Q} 2 \mathrm{D}}\left(k, k^{\prime}\right)=L_{\mathrm{Q} 2 \mathrm{D}}\left(k^{\prime}, k\right)$ for all $k$ and $k^{\prime}$. Furthermore, if $k \neq 0$ and $k^{\prime} \neq 0$, then $L_{\mathrm{Q} 2 \mathrm{D}}\left(k, k^{\prime}\right)=-L_{\mathrm{Q} 2 \mathrm{D}}\left(k,-k^{\prime}\right)$. These results mean that only the lattice summations $L_{\mathrm{Q} 2 \mathrm{D}}\left(0, k^{\prime}\right)$ contribute as all other combinations cancel when summed. These values are $L_{\mathrm{Q} 2 \mathrm{D}}(0,0)=20.402, L_{\mathrm{Q} 2 \mathrm{D}}(0,1)=-0.740$, and $L_{\mathrm{Q} 2 \mathrm{D}}(0,2)=-0.001$ where the number of significant figures reflects the accuracy of the calculation. Note that the rapid convergence means that $L_{\mathrm{Q} 2 \mathrm{D}}$ can be determined for an arbitrary number of layers to good accuracy with just these three results.

The total lattice summation for a $n$-layer Q2D pore can easily be shown to take the following form:

$$
\begin{aligned}
L_{\mathrm{Q} 2 \mathrm{D}, n}= & \frac{1}{n^{2}}\left[n^{2} L_{\mathrm{Q} 2 \mathrm{D}}(0,0)+4 n(n-1) L_{\mathrm{Q} 2 \mathrm{D}}(0,1)\right. \\
& \left.+4 n(n-2) L_{\mathrm{Q} 2 \mathrm{D}}(0,2) \ldots\right] .
\end{aligned}
$$

The results confirm that $L_{\mathrm{Q} 2 \mathrm{D}, n=1}=L_{2 \mathrm{D}}$. In the limit of a $3 \mathrm{D}$ pore, $n \rightarrow \infty$, and in this case $L_{\mathrm{Q} 2 \mathrm{D}, n \rightarrow \infty} \rightarrow L_{\mathrm{Q} 2 \mathrm{D}}(0,0)+$ $4 L_{\mathrm{Q} 2 \mathrm{D}}(0,1)+4 L_{\mathrm{Q} 2 \mathrm{D}}(0,2)+\ldots$, which yields 17.438 . This is in good agreement with the known result $(4 \pi / 3)^{2}$ equal to 17.546 for a spin fluid. The values of $L_{\mathrm{Q} 2 \mathrm{D}}$ for a range of $n$ are presented in Table III.
[1] A. C. A. Muller, K. L. Scrivener, A. M. Gajewicz, and P. J. McDonald, J. Phys. Chem. C 117, 403 (2013).

[2] P. T. Callaghan, Translational Dynamics and Magnetic Resonance (Oxford University Press, Oxford, UK, 2011).

[3] S. Stapf and I. H. S, NMR Imaging in Chemical Engineering (Wiley, New York, 2006).

[4] J. R. Zimmerman and W. E. Brittin, J. Phys. Chem. 61, 1328 (1957).

[5] J.-P. Korb, M. Whaley-Hodges, and R. G. Bryant, Phys. Rev. E 56, 1934 (1997).

[6] A. Abragam, The Principles of Nuclear Magnetism (Oxford University Press, Oxford, UK, 1961).

[7] J.-P. Korb, S. Xu, and J. Jonas, J. Chem. Phys. 98, 2411 (1993).
[8] L. Monteilhet, J.-P. Korb, J. Mitchell, and P. J. McDonald, Phys. Rev. E 74, 061404 (2006).

[9] J.-P. Korb, New J. Phys. 13, 035016 (2011).

[10] R. Valiullin, R. Kimmich, and N. Fatkullin, Phys. Rev. E 56, 4371 (1997).

[11] R. Kimmich, Chem. Phys. 284, 253 (2002).

[12] P. Levitz, M. Zinsmeister, P. Davidson, D. Constantin, and O. Poncelet, Phys. Rev. E 78, 030102 (2008).

[13] O. Bénichou, D. Grebenkov, P. Levitz, C. Loverdo, and R. Voituriez, Phys. Rev. Lett. 105, 150606 (2010).

[14] O. Bénichou, D. Grebenkov, P. Levitz, C. Loverdo, and R. Voituriez, J. Stat. Phys. 142, 657 (2011). 
[15] N. Bloembergen, E. M. Purcell, and R. V. Pound, Phys. Rev. 73, 679 (1948).

[16] H. C. Torrey, Phys. Rev. 92, 962 (1953).

[17] D. A. Faux, D. K. Ross, and C. A. Sholl, J. Phys. C: Solid State Phys. 19, 4115 (1986).

[18] P. J. McDonald, J.-P. Korb, J. Mitchell, and L. Monteilhet, Phys. Rev. E 72, 011409 (2005).

[19] K. R. Brownstein and C. E. Tarr, Phys. Rev. A 19, 2446 (1979).

[20] L. Hua, X. Zhang, and J. M. Titman, J. Phys.: Condens. Matter 9, 9097 (1997).

[21] C. Mattea, H. Tiraboschi, and R. Kimmich, Phys. Rev. E 72, 021602 (2005).

[22] M. Bergenstråhle, J. Wohlert, P. T. Larsson, K. Mazeau, and L. A. Berglund, J. Phys. Chem. B 112, 2590 (2008).

[23] J.-P. Grivet, J. Chem. Phys. 123, 034503 (2005).

[24] C. A. Sholl, J. Phys. C: Solid State Phys. 7, 3378 (1974).

[25] D. Wolf, J. Magn. Reson. 17, 1 (1975).

[26] D. Wolf, J. Phys. C: Solid State Phys. 10, 3545 (1977).

[27] W. A. Barton and C. A. Sholl, J. Phys. C: Solid State Phys. 9, 4315 (1976).

[28] A. Messiah, Quantum Mechanics (North Holland, Amsterdam, 1965)
[29] Z. Romanowski and S. Krukowski, J. Phys. A: Math. Theor. 40, 15071 (2007).

[30] C. A. Sholl, J. Phys. C: Solid State Phys. 14, 447 (1981).

[31] B. R. A. Nijboer and F. W. de Wette, Physica (Amsterdam) 24, 422 (1958).

[32] H. J. C. Berendsen, J. R. Grigera, and T. P. Straatsma, J. Phys. Chem. 91, 6269 (1987).

[33] R. T. Cygan, J.-J. Liang, and A. G. Kalinichev, J. Phys. Chem. B 108, 1255 (2004).

[34] M. P. Allen and D. J. Tildesley, Computer Simulation of Liquids (Oxford University Press, Oxford, UK, 1989).

[35] W. Smith and T. Forester, J. Mol. Graphics 14, 136 (1996).

[36] I. T. Todorov, W. Smith, K. Trachenko, and M. T. Dove, J. Mater. Chem. 16, 1911 (2006).

[37] W. G. Hoover, Phys. Rev. A 31, 1695 (1985).

[38] K. Krynicki, C. D. Green, and D. W. Salter, Faraday Discuss. Chem. Soc. 66, 199 (1978).

[39] I.-C. Yeh and G. Hummer, J. Phys. Chem. B 108, 15873 (2004).

[40] S. Kerisit and C. Liu, Env. Sci. Technol. 43, 777 (2009).

[41] R. A. Tahir-Kheli and R. J. Elliott, Phys. Rev. B 27, 844 (1983).

[42] J. C. Hindman, A. Svirmickas, and M. Wood, J. Chem. Phys. 59, 1517 (1973). 